\title{
Interplay between Locally Excited and Charge Transfer States Governs the Photoswitching Mechanism in the Fluorescent Protein Dreiklang: \\ Supplemental Information
}

Tirthendu Sen ${ }^{a}$, Yingying $\mathrm{Ma}^{a}$, Igor V. Polyakov ${ }^{b, c}$, Bella

L. Grigorenko ${ }^{b, c}$, Alexander V. Nemukhin ${ }^{b, c}$, Anna I. Krylov ${ }^{a}$

${ }^{a}$ Department of Chemistry, University of Southern California, Los Angeles, California 90089-0482

${ }^{b}$ Department of Chemistry, Lomonosov Moscow State University, Moscow, Russia

${ }^{c}$ Emanuel Institute of Biochemical Physics, Russian Academy of Sciences, Moscow, Russia

\section{DEFINITIONS OF PROTONATION STATES}
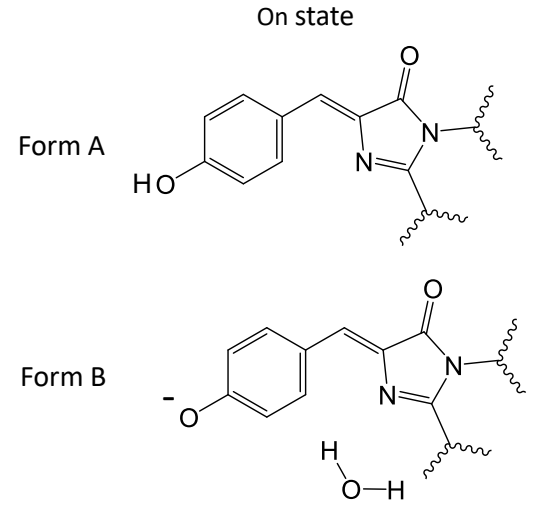
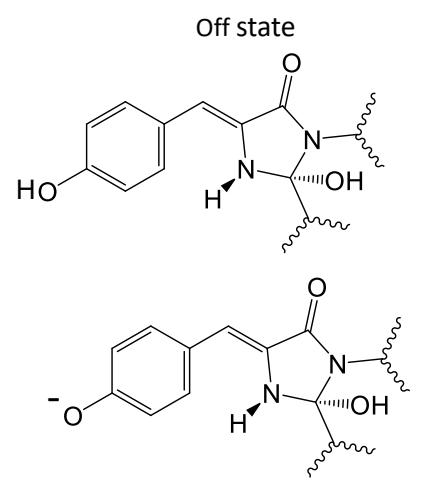

FIG. S1: Definition of chromophore states. 


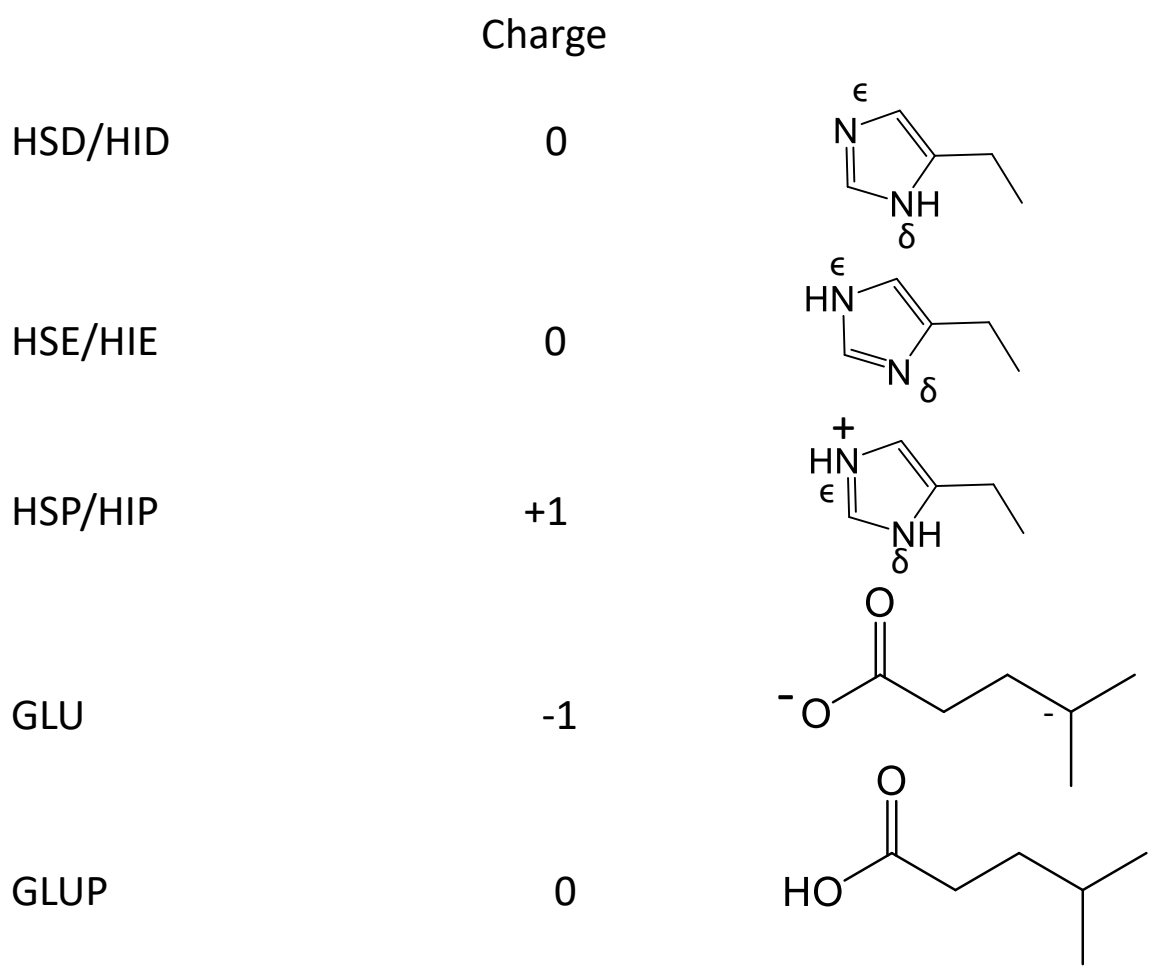

FIG. S2: Definition of protonation states of Glu222 and His145 in Dreiklang. GLUP can exist in two conformations: As shown or protonated on the other oxygen (GLUP2). 


\section{COMPUTATIONAL DETAILS}

In addition to the structures obtained by the computational protocol described in the main manuscript, we also consider the structures from our previous study ${ }^{1}$ in which we started with 3ST2 structure and used QM/MM optimization with electrostatic embedding, as implemented in NWChem. The QM part was described by M06-L/cc-pVDZ and the MM part was described by the $A M B E R$ forcefield ${ }^{2}$. In these calculations ${ }^{1}$, QM included the chromophore, side chains of Gln94, Arg96, His145, Tyr203, Ser205, and Glu222, and seven water molecules. This definition is similar to our extended QM. We note that these model structures also included additional water molecule, which is present in 3ST3 structure (OFF-state) but not seen in 3ST2 and 3ST4. The comparisons between the two protocols quantify the effect of the level of theoretical treatment.

The key structural parameters two sets of structures are compared in Tables S7-S12 below and graphically in Figs. S4 and S5.

\section{FORCEFIELD PARAMETERS FOR THE NEUTRAL HYDRATED CHROMOPHORE}

To derive missing forcefield parameters (for the OFF-form of the chromophore) we followed a protocol described in our previous work ${ }^{3,4}$. The key equations and the values of the forcefield parameters are given below.

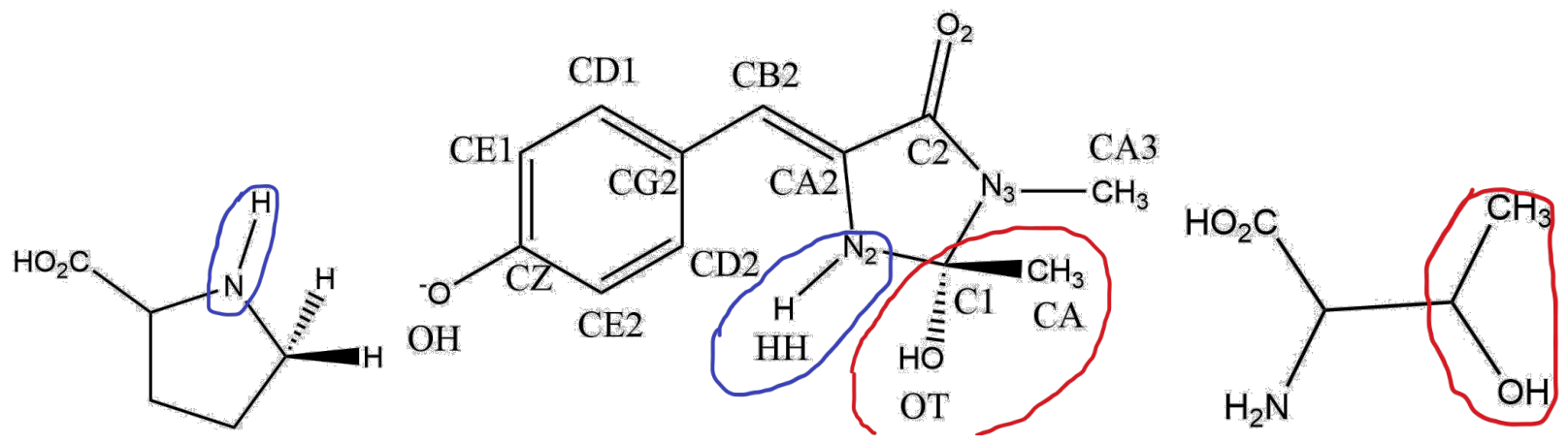

FIG. S3: From left to right: proline, chromophore in off-state, threonine.

$$
\Delta q_{(\text {on,charmm-qm })}=q_{o n, \text { charmm }}-q_{N B O(o n, q m)}
$$




$$
q_{(o f f, c h a r m m)}=q_{N B O(o f f, q m)}+\Delta q_{(o n, c h a r m m-q m)}
$$

$$
\begin{gathered}
E=k\left(b-b_{0}\right)^{2} \\
k_{\text {off,param }}=\frac{k_{\text {off,theory }}}{k_{\text {on,theory }}} \times k_{\text {on-charmm }}
\end{gathered}
$$

$b_{0}$ is the equilibrium bond length.

$$
\begin{gathered}
E=k\left(A-A_{0}\right)^{2} \\
k_{\text {off,param }}=\frac{k_{\text {off,theory }}}{k_{\text {on,theory }}} \times k_{\text {on-charmm }}
\end{gathered}
$$

$A_{0}$ is the equilibrium bond angle.

$$
E=k[1+\cos (n \phi-\delta)]
$$

where $n$ is the phase, $\delta$ is the optimized dihedral angle.

$$
k_{\text {off,param }}=\frac{k_{\text {off,theory }}}{k_{\text {on,theory }}} \times k_{\text {on-charmm }}
$$


TABLE S1: Partial charges in the OFF-state.

\begin{tabular}{|c|c|c|c|c|c|c|}
\hline Atom, off & on, charmm & on, qm & $\Delta q_{(\text {on }, \text { charmm-qm })}$ & $\Delta q_{\text {adjusted }}$ & off, qm & off, charmm \\
\hline C1(threonine) & 0.10 & 0.15 & -0.05 & -0.02 & 0.67 & 0.69 \\
\hline N2 (proline) & -0.74 & -0.28 & -0.46 & -0.43 & -0.68 & -0.25 \\
\hline N3 & -0.64 & -0.52 & -0.12 & -0.09 & -0.56 & -0.47 \\
\hline $\mathrm{C} 2$ & 0.8 & 0.74 & 0.06 & 0.09 & 0.74 & 0.65 \\
\hline $\mathrm{O} 2$ & -0.61 & -0.60 & -0.01 & 0.02 & -0.63 & -0.65 \\
\hline $\mathrm{CA} 2$ & 0.24 & 0.05 & 0.19 & 0.22 & 0.12 & -0.10 \\
\hline CB2 & -0.10 & -0.09 & -0.01 & 0.02 & -0.24 & -0.26 \\
\hline HB2 & 0.1 & 0.28 & -0.18 & -0.15 & 0.27 & 0.42 \\
\hline CG2 & 0.00 & -0.11 & 0.11 & 0.14 & -0.09 & -0.23 \\
\hline CD1 & -0.115 & -0.14 & 0.025 & 0.06 & -0.185 & -0.245 \\
\hline HD11 & 0.115 & 0.27 & -0.155 & -0.12 & 0.25 & 0.37 \\
\hline CD2 & -0.115 & -0.14 & 0.025 & 0.06 & -0.185 & -0.245 \\
\hline HD21 & 0.115 & 0.27 & -0.155 & -0.12 & 0.25 & 0.37 \\
\hline CE1 & -0.115 & -0.27 & 0.155 & 0.19 & -0.275 & -0.465 \\
\hline HE11 & 0.115 & 0.27 & -0.155 & -0.12 & 0.25 & 0.37 \\
\hline CE2 & -0.115 & -0.27 & 0.155 & 0.19 & -0.275 & -0.465 \\
\hline HE21 & 0.115 & 0.27 & -0.155 & -0.12 & 0.25 & 0.37 \\
\hline $\mathrm{CZ}$ & 0.11 & 0.38 & -0.27 & -0.24 & 0.34 & 0.58 \\
\hline $\mathrm{OH}$ & -0.54 & -0.68 & 0.14 & 0.17 & -0.70 & -0.87 \\
\hline $\mathrm{OHH}$ & 0.43 & 0.52 & -0.09 & -0.06 & 0.50 & 0.56 \\
\hline OT (threonine) & -0.78 & -0.65 & -0.13 & -0.10 & -0.75 & -0.65 \\
\hline HT(threonine) & 0.50 & 0.44 & 0.06 & 0.09 & 0.50 & 0.41 \\
\hline HH (proline) & 0.41 & 0.11 & 0.30 & 0.33 & 0.44 & 0.11 \\
\hline CA3 & & -0.18 & & & & -0.18 \\
\hline HA31 & & 0.09 & & & & 0.09 \\
\hline HA32 & & 0.09 & & & & 0.09 \\
\hline $\mathrm{C}$ & & 0.51 & - & - & - & 0.51 \\
\hline $\mathrm{O}$ & & $-0.51-$ & - & - & - & -0.51 \\
\hline $\mathrm{N}$ & & $-0.47-$ & - & - & - & -0.47 \\
\hline $\mathrm{HN}$ & & $0.31-$ & - & - & - & 0.31 \\
\hline $\mathrm{CA}$ & & -0.02 & & & & -0.02 \\
\hline HA1 & & 0.09 & & & & 0.09 \\
\hline HA2 & & 0.09 & & & & 0.09 \\
\hline
\end{tabular}


TABLE S2: Optimized bond lengths (in $\AA$ ) involving key atoms.

\begin{tabular}{|l|l|l|l|}
\hline Bonds & $B_{\text {on,charmm }}$ & $B_{\text {on }, \text { opt }}$ & $B_{\text {off,opt }}$ \\
\hline C1-N2 (proline) & 1.434 & 1.46 & 1.45 \\
N2-CA2 & 1.40 & 1.41 & 1.39 \\
N2-HH (proline) & 0.997 & 1.02 & 1.01 \\
C1-OT (threonine) & 1.42 & 1.40 & 1.41 \\
C1-CA & 1.49 & 1.49 & 1.53 \\
C1-N3 & 1.39 & 1.38 & 1.45 \\
\hline
\end{tabular}

TABLE S3: Parameterization of the force constant $k$ for bond lengths in $\mathrm{kcal} / \mathrm{mol} / \AA^{2}$.

\begin{tabular}{|l|l|l|l|l|l|}
\hline Bonds & $k_{\text {on,charmm }}$ & $k_{\text {on,theory }}$ & $k_{\text {off,theory }}$ & $k_{\text {off,theory }} / k_{\text {on }, \text { theory }}$ & $k_{\text {off,param }}$ \\
\hline C1-N2 (proline) & 320 & 1156.50 & 1226.15 & 1.06 & 339.27 \\
N2-CA2 & 400 & 940.00 & 1257.53 & 1.34 & 535.12 \\
N2-HH (proline) & 440 & 976.40 & 1044.80 & 1.07 & 470.82 \\
C1-OT (threonine) & 428 & 847.14 & 744.22 & 0.88 & 376.00 \\
C1-CA & 354 & 562.25 & 542.79 & 0.965 & 341.75 \\
C1-N3 & 320 & 1156.50 & 1226.15 & 1.06 & 339.27 \\
\hline
\end{tabular}

TABLE S4: Optimized bond angles (in degrees) involving key atoms.

\begin{tabular}{|l|l|l|l|}
\hline Angles & $A_{\text {on,charmm }}$ & $A_{\text {on,opt }}$ & $A_{\text {off,opt }}$ \\
\hline N2-C1-N3 & 114.0 & 113.99 & 102.31 \\
C1-N2-CA2 & 106.0 & 106.18 & 111.21 \\
HH-N2-C1 (proline) & 117.0 & 111.44 & 115.90 \\
HH-N2-CA2 & 117.0 & 111.44 & 117.8 \\
OT-C1-CA (threonine) & 110.1 & 112.6 & 110.31 \\
OT-C1-N2 (threonine) & 110.1 & 112.6 & 107.90 \\
OT-C1-N3 (threonine) & 110.1 & 112.6 & 111.39 \\
N2-CA2-CB2 & 129.5 & 129.58 & 130.17 \\
N2-CA2-C2 & 108.3 & 108.73 & 106.54 \\
C1-N3-C2 & 107.9 & 113.47 & 108.26 \\
CA-C1-N3 (threonine) & 113.5 & 111.6 & 112.01 \\
\hline
\end{tabular}


TABLE S5: Parameterization of the force constant $k$ for bond angles in $\mathrm{kcal} / \mathrm{mol} / \mathrm{rad}^{\circ 2}$.

\begin{tabular}{|l|l|l|l|l|l|}
\hline Angles & $k_{\text {on, charmm }}$ & $k_{\text {on,theory }}$ & $k_{\text {off,theory }}$ & $k_{\text {off,theory }} / k_{\text {on }, \text { theory }}$ & $k_{\text {off,param }}$ \\
\hline N2-C1-N3 & 130.0 & 444.28 & 347.33 & 0.78 & 101.6 \\
C1-N2-CA2 & 130.0 & 438.63 & 259.98 & 0.59 & 77.05 \\
HH-N2-C1 (proline) & 35.0 & 89.73 & 79.69 & 0.89 & 31.08 \\
HH-N2-CA2 & 35.0 & 89.73 & 79.69 & 0.89 & 31.08 \\
OT-C1-CA (threonine) & 75.7 & 232.18 & 259.37 & 1.12 & 84.56 \\
OT-C1-N2(N3) (threonine) & 75.7 & 232.18 & 259.37 & 112 & 84.56 \\
N2-CA2-CB2 & 45.8 & 151.23 & 169.42 & 1.12 & 51.3 \\
N2-CA2-C2 & 130.0 & 472.5 & 376.50 & 0.797 & 103.6 \\
C1-N3-C2 & 130.0 & 498.24 & 305.6 & 0.61 & 79.7 \\
CA-C1-N3 (threonine) & 70.0 & 180.72 & 179.47 & 0.99 & 69.5 \\
\hline
\end{tabular}

TABLE S6: Parameterization of the force constant $k$ for dihedral angles; $\delta$ in degrees, $k$ in kcal $/ \mathrm{mol}$.

\begin{tabular}{|l|l|l|l|l|l|l|}
\hline Angles & $k_{\text {on, charmm }}$ & $\mathrm{n}$ & $\delta$ & $k_{\text {off,theory }} / k_{\text {on }, \text { theory }}$ & $k_{\text {off,param }}$ \\
\hline OT-C1-N2-HH & 0.16 & 3 & 180 & 0.263 & 0.053 \\
OT-C1-N2-CA2 & 0.20 & 3 & 0 & 13.83 & 2.213 \\
CA-C1-N2-HH & 0.16 & 3 & 180 & 0.263 & 0.053 \\
HH-N2-CA2-CB2 & 0.16 & 3 & 0 & 0.263 & 0.053 \\
HH-N2-CA2-C2 & 0.20 & 3 & 180 & 13.83 & 2.213 \\
HH-N2-C1-N3 & 0.20 & 3 & 180 & 13.83 & 2.213 \\
CA-C1-N3-CA3 & 0.16 & 3 & 0 & 0.263 & 0.053 \\
OT-C1-N3-CA3 & 0.16 & 3 & 0 & 0.263 & 0.053 \\
CA-C1-N3-C2 & 0.20 & 3 & 180 & 13.83 & 2.213 \\
\hline
\end{tabular}




\section{STRUCTURES OF MODEL SYSTEMS}

TABLE S7: Comparison of the distances (in $\AA$ ) from MD and QM/MM simulations with crystal structure 3ST4 (ON-state). The chromophore is neutral (A-form). 'md' denotes structures averaged over equilibrium MD trajectories. 'opt' and 'opt2' denote the QM/MM optimized structures obtained with present protocol and with the protocol from Ref. 1, respectively.

\begin{tabular}{|c|c|c|c|c|c|c|c|c|c|c|c|c|}
\hline D & & $3 \mathrm{ST} 4$ & $\begin{array}{l}\text { HSE- } \\
\text { GLU }\end{array}$ & $\Delta$ & $\begin{array}{l}\text { HSE- } \\
\text { GLUP }\end{array}$ & $\Delta$ & $\begin{array}{l}\text { HSD- } \\
\text { GLU }\end{array}$ & $\Delta$ & $\begin{array}{l}\text { HSD- } \\
\text { GLUP }\end{array}$ & $\Delta$ & $\begin{array}{l}\text { HSP- } \\
\text { GLU }\end{array}$ & $\Delta$ \\
\hline \multirow[t]{3}{*}{ d1 } & & 3. & $3.42(0.3)$ & 0.10 & 3.96( & 0.44 & $3.74(0.4)$ & 0.22 & 3.63 & 0.11 & 68 & .1 \\
\hline & & - & 3.31 & 0.21 & 3.92 & 0.4 & 3.53 & 0.01 & 4.2 & 68 & 3.78 & 1.2 \\
\hline & $\mathrm{t} 2$ & - & 3.45 & 0.07 & 3.50 & 0.02 & 3.49 & 0.03 & & & .46 & .0 \\
\hline \multirow[t]{3}{*}{$\mathrm{d} 2$} & $\mathrm{~d}$ & 2.97 & 3.94( & 0.97 & 3.44( & 0.47 & 3.92( & 0.95 & 3.54( & 0.57 & 4.00 & 10 \\
\hline & t & - & 3.99 & 1.02 & 2.78 & 0.19 & 4.06 & 1.09 & 2.86 & 0.11 & 4.00 & 1.0 \\
\hline & pt2 & - & 3.46 & 0.49 & 3.09 & 0.12 & 3.44 & 0.47 & & & 3.49 & 0.5 \\
\hline \multirow[t]{3}{*}{$\mathrm{d} 3$} & $\mathrm{id}$ & 2.73 & 3.42( & 0.69 & $2.77(0.1)$ & 0.04 & 2.74( & 0.01 & $2.77(0.1)$ & 0.04 & 2.74( & 0.0 \\
\hline & pt & - & 2.58 & 0.15 & 2.62 & 0.11 & 2.6 & 0.13 & 2.67 & 0.06 & 2.61 & 0.1 \\
\hline & pt2 & - & 2.77 & 0.04 & 2.85 & 0.12 & 2.80 & 0.07 & & & 2.81 & 0.0 \\
\hline \multirow[t]{3}{*}{$\mathrm{d} 4$} & $\mathrm{~d}$ & 3.81 & 3.60 & 21 & 4.82( & 1.01 & 3.62 & 0.19 & & 0.10 & $4.02(0$ & 0. \\
\hline & pt & - & 3.53 & 0.28 & 3.67 & 0.14 & 3.52 & 0.29 & 3.77 & 0.04 & 3.38 & 0.4 \\
\hline & opt2 & - & 3.96 & 0.15 & 3.92 & 0.11 & 3.96 & 0.36 & & & 4.16 & 0.3 \\
\hline \multirow[t]{3}{*}{$\mathrm{d} 5$} & $\mathrm{id}$ & 4.04 & 4.38 & 0.34 & 4.08 & 0.04 & 4.05 & 0.01 & 3.86 & 0.18 & 4.67 & 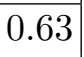 \\
\hline & $\mathrm{t}$ & - & - & - & - & - & - & - & - & - & - & - \\
\hline & pt2 & - & - & - & - & - & - & - & - & - & - & - \\
\hline \multirow[t]{3}{*}{$\mathrm{d} 6$} & d & 3.64 & 3.85( & 21 & $3.82(0.2)$ & 0.18 & 3.88 & 0.24 & 3.87 & 0.23 & $3.82(0$ & 0.1 \\
\hline & 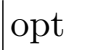 & - & 3.69 & 0.05 & 3.52 & 0.12 & 3.63 & 0.01 & 3.6 & 0.04 & 3.59 & 0.0 \\
\hline & opt2 & - & 3.85 & 0.21 & 3.63 & 0.01 & 3.68 & 0.04 & & & 3.67 & 0.0 \\
\hline \multirow[t]{3}{*}{$\mathrm{d} 7$} & dd & 2.6 & V.L & 4 & $2.69(0$ & 0.06 & 2.71( & 0.08 & 2.74( & 0.11 & 3.19( & 0. \\
\hline & opt & - & 2.97 & 0.34 & 2.59 & 0.04 & 2.63 & 0 & 2.63 & 0 & 3.59 & 0.1 \\
\hline & opt2 & - & 2.59 & 0.04 & 2.66 & 0.03 & 2.66 & 0.03 & & & 2.67 & 0.0 \\
\hline \multirow[t]{3}{*}{$\mathrm{d} 8$} & md & 3.41 & $3.18(6$ & 0.23 & 3.43( & 0.02 & 3.49 ( & 0.08 & $3.43(0.2)$ & 0.02 & $3.6(0$ & .1 \\
\hline & opt & - & 3.25 & 0.16 & 3.22 & 0.19 & 3.71 & 0.3 & 3.27 & 0.14 & 3.59 & 0.1 \\
\hline & opt2 & - & 3.05 & 0.36 & 3.26 & 0.15 & 3.02 & 0.39 & & & 3.01 & 0.4 \\
\hline \multirow[t]{3}{*}{$\mathrm{d} 9$} & md & 2.89 & 2.92( & 0.03 & 2.99 & 0.10 & 2.88( & 0.01 & 2.99 & 0.10 & 2.96 & 0.0 \\
\hline & opt & - & 2.64 & 0.25 & 2.68 & 0.21 & 2.78 & 0.11 & 2.67 & 0.22 & 2.79 & 0.1 \\
\hline & opt2 & - & 2.82 & 0.07 & 2.81 & 0.08 & 2.79 & 0.10 & & & 2.81 & 0.0 \\
\hline \multirow[t]{3}{*}{ d10 } & id & 2.69 & $2.63(0.1)$ & 0.06 & $2.81(0.2)$ & 0.12 & $2.63(0.1)$ & 0.06 & $2.78(0.2)$ & 0.09 & $2.66(0.1)$ & 0.0 \\
\hline & opt & - & 2.58 & 0.11 & 2.7 & 0.01 & 2.8 & 0.11 & 2.72 & 0.03 & 2.73 & 0.0 \\
\hline & pt2 & - & 2.67 & 0.02 & 2.75 & 0.06 & 2.67 & 0.02 & & & 2.67 & 0.0 \\
\hline
\end{tabular}


TABLE S8: Comparison of the distances (in $\AA$ ) from MD and QM/MM simulations with crystal structure 3ST4 (ON-state). The chromophore is neutral (A-form). 'md' denotes structures averaged over equilibrium MD trajectories. 'opt' and 'opt2' denote the QM/MM optimized structures obtained with present protocol and with the protocol from Ref. 1, respectively.

\begin{tabular}{|l|l|l|l|l|l|l|l|l|l|l|l|l|}
\hline D & & $3 S T 4$ & HSE- & $\Delta$ & HSE- & $\Delta$ & HSD- & $\Delta$ & HSD- & $\Delta$ & HSP- & $\Delta$ \\
GLU & & & GLUP & & GLU & & GLUP & & GLU & \\
\hline $\mathrm{d} 11$ & md & 2.73 & V.L & - & $3.76(0.8)$ & 1.03 & $2.9(0.2)$ & 0.17 & $3.02(0.3)$ & 0.29 & $2.93(0.3)$ & 0.20 \\
& opt & - & 3.01 & 0.28 & 2.7 & 0.03 & 2.52 & 0.21 & 2.59 & 0.14 & 2.89 & 0.16 \\
& opt2 & - & 2.72 & 0.01 & 2.69 & 0.04 & 2.67 & 0.06 & & & 2.63 & 0.10 \\
\hline $\mathrm{d} 12$ & $\mathrm{md}$ & 3.05 & V.L & - & $3.06(0.3)$ & 0.01 & $3.53(0.4)$ & 0.48 & $3.42(0.4)$ & 0.37 & $3.89(0.4)$ & 0.84 \\
& opt & - & 3.33 & 0.28 & 2.58 & 0.47 & 2.83 & 0.22 & 2.95 & 0.1 & 3.23 & 0.18 \\
& opt2 & - & 3.53 & 0.48 & 2.70 & 0.35 & 3.10 & 0.05 & & & 2.74 & 0.31 \\
\hline $\mathrm{d} 13$ & md & 2.89 & V.L & - & $2.84(0.3)$ & 0.05 & $3.4(0.4)$ & 0.51 & $3.41(0.6)$ & 0.52 & $3.87(0.4)$ & 0.98 \\
& opt & - & - & - & - & - & - & - & - & - & - & - \\
& opt2 & - & - & - & - & - & - & - & - & - & - & - \\
\hline $\mathrm{d} 14$ & md & 4.18 & $2.67(0.1)$ & 1.51 & $4.28(0.5)$ & 0.10 & $2.66(0.1)$ & 1.52 & $3.48(0.4)$ & 0.70 & $2.68(0.1)$ & 1.5 \\
& opt & - & 2.7 & 1.48 & 4.72 & 0.54 & 2.56 & 1.62 & 4.32 & 0.14 & 2.5 & 1.68 \\
& opt2 & - & 5.06 & 0.88 & 4.38 & 0.30 & 5.02 & 0.84 & & & 5.03 & 0.85 \\
\hline
\end{tabular}


TABLE S9: Comparison of the distances (in $\AA$ ) from MD and QM/MM simulations with crystal structure 3ST4 (ON-state). Chromophore is anionic (B-form). 'md' denotes structures averaged over equilibrium MD trajectories. 'opt' and 'opt2' denote the QM/MM optimized structures obtained with present protocol and with the protocol from Ref. 1, respectively.

\begin{tabular}{|c|c|c|c|c|c|c|c|c|c|c|c|c|}
\hline $\mathrm{D}$ & & ST4 & $\begin{array}{l}\text { HSE- } \\
\text { GLU }\end{array}$ & $\Delta$ & $\begin{array}{l}\text { HSE- } \\
\text { GLUP }\end{array}$ & $\Delta$ & $\begin{array}{l}\text { HSD- } \\
\text { GLU }\end{array}$ & $\Delta$ & $\begin{array}{l}\text { HSD- } \\
\text { GLUP }\end{array}$ & $\Delta$ & $\begin{array}{l}\text { HSP- } \\
\text { GLUP }\end{array}$ & $\Delta$ \\
\hline \multirow[t]{3}{*}{ d1 } & & 3.52 & $3.90(0.3)$ & 0.38 & $3.19(0.2)$ & 0.33 & $3.57(0.4)$ & 0.05 & $3.32(0.3)$ & 0.20 & 3.05( & 0.4 \\
\hline & & - & 3.66 & 0.14 & 3.39 & 0.13 & 3.39 & 0.13 & 3.47 & 0.05 & 2.88 & 0.64 \\
\hline & t2 & - & & & 3.30 & 0.22 & & & 3.24 & 0.28 & 3.10 & 0.4 \\
\hline \multirow[t]{3}{*}{$\mathrm{d} 2$} & $\mathrm{~d}$ & 2.97 & 3) & 01 & 3.17 & 0.20 & 4) & 1.65 & $3.13(0.2)$ & 0.16 & $3.07(0.2)$ & 0.1 \\
\hline & 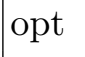 & - & 4.02 & 1.05 & 2.74 & 0.23 & 4.11 & 1.14 & 2.9 & 0.07 & 2.79 & 0.1 \\
\hline & opt2 & - & & & 3.47 & 0.50 & & & 3.49 & 0.52 & 3.08 & 0.1 \\
\hline \multirow[t]{3}{*}{$\mathrm{d} 3$} & $\bar{d}$ & 2.73 & 2.72( & 0.01 & 2.75 & 0.02 & 2.74 & 0.01 & 2.72( & .04 & $2.73(0.1)$ & .0 \\
\hline & opt & - & 2.52 & 0.21 & 2.57 & 0.16 & 2.51 & 0.22 & 2.57 & 0.16 & 2.64 & 0 \\
\hline & opt2 & - & & & 2.74 & 0.01 & & & 2.76 & 0.03 & 2.81 & 0.0 \\
\hline \multirow[t]{3}{*}{$\mathrm{d} 4$} & $\mathrm{~d}$ & 3.81 & ) & 33 & 4.83 & 1.02 & $3.7:$ & 0.08 & 3.97 & 0.16 & 4.42 & .61 \\
\hline & opt & - & 3.93 & 0.12 & 3.84 & 0.03 & 3.33 & 0.48 & 4.06 & 0.25 & 4.51 & 0.7 \\
\hline & opt2 & - & & & 4.01 & 0.20 & & & 4.01 & 0.20 & 3.99 & 0.1 \\
\hline \multirow[t]{3}{*}{$\mathrm{d} 5$} & d & 4.04 & 4.22 & 0.18 & 4.74 & 0.70 & 4.15 & 0.11 & 3.82 & 0.22 & 4.14 & 0.1 \\
\hline & opt & - & - & 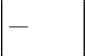 & - & - & - & - & - & - & - & - \\
\hline & opt2 & - & - & - & - & - & - & - & - & - & - & - \\
\hline \multirow[t]{3}{*}{ d6 } & $d$ & 3.64 & 3.96 & 0.32 & $3.78(0$ & 0.14 & 4.00 & 0.36 & $3.84(0.2)$ & .20 & $3.94(0$ & 0.3 \\
\hline & opt & - & 3.84 & 0.20 & 3.64 & 0.0 & 3.97 & 0.33 & 3.67 & 0.03 & 3.64 & 0.0 \\
\hline & opt2 & - & & & 3.68 & 0.04 & & & 3.83 & 0.19 & 3.68 & 0.0 \\
\hline \multirow[t]{3}{*}{$\mathrm{d} 7$} & md & 2.63 & $2.79(0.2)$ & 0 & 5.24( & 2.61 & $2.8(0$ & 0.17 & $2.75(0.2)$ & 0.12 & $2.79(\mathrm{C}$ & 0.1 \\
\hline & opt & - & 2.86 & 0.23 & 5.14 & 2.51 & 2.87 & 0.24 & 2.59 & 0.04 & 2.54 & 0.0 \\
\hline & opt2 & - & & & 2.74 & 0.11 & & & 2.68 & 0.05 & 2.63 & 0.0 \\
\hline \multirow[t]{3}{*}{$\mathrm{d} 8$} & md & 3.4 & .00( & 0.41 & 3.86( & 0.45 & I. & 0.40 & 3.72( & 0.31 & 3.88( & $0.4^{\prime}$ \\
\hline & opt & - & 3.19 & 0.22 & 3.29 & 0.12 & 3.47 & 0.06 & 3.23 & 0.18 & 3.51 & 0.1 \\
\hline & opt2 & - & & & 3.34 & 0.07 & & & 3.02 & 0.39 & 3.27 & 0.1 \\
\hline \multirow[t]{3}{*}{$\mathrm{d} 9$} & md & 2.89 & $2.83(0.1)$ & .06 & $2.86(0.2)$ & 0.03 & $2.84(0.1)$ & 0.05 & $2.84(0.1)$ & 0.05 & $2.85(0.2)$ & 0.0 \\
\hline & opt & - & 2.71 & 0.18 & 2.88 & 0.01 & 2.58 & 0.41 & 2.77 & 0.12 & 2.77 & 0.1 \\
\hline & opt2 & - & & & 2.87 & 0.02 & & & 2.86 & 0.03 & 2.80 & 0.0 \\
\hline \multirow[t]{3}{*}{$\mathrm{d} 10$} & $\mathrm{nd}$ & 2.69 & $2.66(0.1)$ & 3 & $2.8(0.2)$ & 0.11 & $2.64(0.1)$ & 0.05 & $2.78(0.2)$ & 0.09 & $2.78(0.2)$ & 0.0 \\
\hline & opt & - & 2.46 & 0.23 & 2.64 & 0.05 & 2.89 & 0.20 & 2.77 & 0.08 & 2.81 & 0.1 \\
\hline & opt2 & - & & & 2.91 & 0.22 & & & 2.89 & 0.20 & 2.75 & 0.0 \\
\hline
\end{tabular}


TABLE S10: Comparison of the distances (in $\AA$ ) from MD and QM/MM simulations with crystal structure 3ST4 (ON-state). Chromophore is anionic (B-form). 'md' denotes structures averaged over equilibrium MD trajectories. 'opt' and 'opt2' denote the QM/MM optimized structures obtained with present protocol and with the protocol from Ref. 1, respectively.

\begin{tabular}{|l|l|l|l|l|l|l|l|l|l|l|l|l|}
\hline D & & $3 S T 4$ & $\begin{array}{l}\text { HSE- } \\
\text { GLU }\end{array}$ & $\Delta$ & $\begin{array}{l}\text { HSE- } \\
\text { GLUP }\end{array}$ & $\Delta$ & $\begin{array}{l}\text { HSD- } \\
\text { GLU }\end{array}$ & $\Delta$ & $\begin{array}{l}\text { HSD- } \\
\text { GLUP }\end{array}$ & $\Delta$ & $\begin{array}{l}\text { HSP- } \\
\text { GLUP }\end{array}$ & $\Delta$ \\
\hline $\mathrm{d} 11$ & md & 2.73 & $3.67(0.7)$ & 0.94 & $4.54(0.8)$ & 1.81 & $2.9(0.2)$ & 0.17 & $2.9(0.2)$ & 0.17 & $2.97(0.4)$ & 0.24 \\
& opt & - & 3.95 & 1.22 & 4.11 & 1.38 & 2.66 & 0.07 & 2.74 & 0.01 & 2.76 & 0.03 \\
& opt2 & - & & & 2.62 & 0.11 & & & 2.63 & 0.10 & 2.70 & 0.03 \\
\hline $\mathrm{d} 12$ & $\mathrm{md}$ & 3.05 & $5.41(0.3)$ & 2.36 & $3.07(0.4)$ & 0.02 & $3.43(0.4)$ & 0.38 & $3.25(0.3)$ & 0.20 & $3.33(0.3)$ & 0.28 \\
& opt & - & 3.31 & 0.26 & 2.89 & 0.16 & 3.76 & 0.71 & 2.85 & 0.2 & 2.65 & 0.40 \\
& opt2 & - & & & 3.37 & 0.32 & & & 3.31 & 0.26 & 2.76 & 0.29 \\
\hline $\mathrm{d} 13$ & md & 2.89 & $3.38(0.6)$ & 0.49 & $4.73(0.5)$ & 1.84 & $3.25(0.3)$ & 0.36 & $2.81(0.2)$ & 0.08 & $3.24(0.5)$ & 0.35 \\
& opt & - & - & - & - & - & - & - & - & - & - & - \\
& opt2 & - & - & - & - & - & - & - & - & - & - & - \\
\hline $\mathrm{d} 14$ & md & 4.18 & $2.68(0.1)$ & 1.50 & $4.33(0.5)$ & 0.15 & $2.65(0.1)$ & 1.53 & $4.1(0.4)$ & 0.08 & $5.24(0.4)$ & 1.06 \\
& opt & - & 2.65 & 1.53 & 4.27 & 0.09 & 2.55 & 1.63 & 4.59 & 0.41 & 5.79 & 1.61 \\
& opt2 & - & & & 4.90 & 0.72 & & & 4.99 & 0.81 & 4.53 & 0.35 \\
\hline
\end{tabular}


TABLE S11: Comparison of the distances (in $\AA$ ) from MD and QM/MM simulations with crystal structure 3ST3 (OFF-state). Chromophore is neutral. 'md' denotes structures averaged over equilibrium MD trajectories. 'opt' and 'opt2' denote the QM/MM optimized structures obtained with present protocol and with the protocol from Ref. 1, respectively.

\begin{tabular}{|c|c|c|c|c|c|c|c|c|c|c|c|c|c|c|c|c|}
\hline D & & ST3 & $\begin{array}{l}\text { HSE } \\
\text { GLU }\end{array}$ & $\Delta$ & \begin{tabular}{|l|} 
HSD \\
GLU \\
$\mathrm{P}$ \\
\end{tabular} & $\Delta$ & $\begin{array}{l}\text { HSE } \\
\text { GLU } \\
\text { P2 } \\
\end{array}$ & $\Delta$ & \begin{tabular}{|l|} 
HSD \\
GLU \\
P2 \\
\end{tabular} & $\Delta$ & $\begin{array}{l}\text { HSE } \\
\text { GLU }\end{array}$ & $\Delta$ & $\begin{array}{l}\text { HSD } \\
\text { GLU }\end{array}$ & $\Delta$ & $\begin{array}{l}\mathrm{ISP} \\
\mathrm{i} \mathrm{LU}\end{array}$ & $\Delta$ \\
\hline \multirow[t]{3}{*}{$\mathrm{d} 1$} & $\mathrm{~d}$ & 5 & $\begin{array}{l}36 \\
.3)\end{array}$ & 0.0 & $\begin{array}{l}3.75 \\
(0.5)\end{array}$ & 0.4 & $\begin{array}{l}3.71 \\
(0.6)\end{array}$ & 0.3 & $\begin{array}{c}3.68 \\
(0.2)\end{array}$ & 0.3 & $\begin{array}{l}63 \\
.5)\end{array}$ & 3 & $\begin{array}{l}3.53 \\
(0.4)\end{array}$ & .2 & $\begin{array}{l}2.97 \\
(0.2)\end{array}$ & \\
\hline & opt & - & 1 & 0.7 & 2.96 & 0.4 & 3.17 & 0.2 & 2 & 0.8 & 4.29 & 0.9 & .48 & 0.1 & 38 & \\
\hline & opt2 & 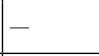 & 3.58 & 0.2 & 3.27 & 0.1 & & & & & 3.44 & .1 & 47 & 0.1 & .46 & \\
\hline \multirow[t]{3}{*}{$\mathrm{d} 2$} & $\mathrm{md}$ & 46 & $\begin{array}{l}3.25 \\
(0.2)\end{array}$ & 0.8 & $\begin{array}{l}3.28 \\
(0.2)\end{array}$ & 0.8 & 4) & .7 & $\begin{array}{l}53 \\
4)\end{array}$ & 2.1 & $\begin{array}{l}20 \\
.2)\end{array}$ & .7 & $\begin{array}{l}23 \\
.2)\end{array}$ & .8 & $\begin{array}{l}13 \\
.1)\end{array}$ & \\
\hline & opt & - & 2.8 & 0.3 & 2.85 & 0.4 & 2.76 & 0.3 & 2.67 & 0.2 & 2.66 & 0.2 & 2.67 & 0.2 & 2.78 & \\
\hline & opt2 & - & 82 & 0.4 & 2.86 & 0.4 & & & & & .62 & .2 & 2.63 & 0.2 & 2.63 & \\
\hline \multirow[t]{3}{*}{ d3 } & $\mathrm{md}$ & 2.85 & $\begin{array}{l}23 \\
.6)\end{array}$ & 1.4 & $\begin{array}{l}4.32 \\
(0.4)\end{array}$ & 1.5 & $\begin{array}{l}6 \\
3)\end{array}$ & 7 & 3) & 0.6 & $\begin{array}{l}3.91 \\
(0.4)\end{array}$ & .1 & $\begin{array}{l}3.67 \\
(0.5)\end{array}$ & 8 & $\begin{array}{l}51 \\
.3)\end{array}$ & \\
\hline & opt & - & & 0.6 & 3.45 & 0.6 & 13 & 0.3 & $J 9$ & 0.2 & 2.96 & .1 & 3.03 & .2 & & \\
\hline & opt2 & _ & 3.17 & 0.3 & 3.17 & 0.3 & & & & & 2.96 & 0.1 & 2.96 & 0.1 & 2.98 & \\
\hline \multirow[t]{3}{*}{$\mathrm{d} 4$} & $\mathrm{md}$ & 3.01 & 2.69 & 0.3 & $\begin{array}{l}2.7 \\
(0.1)\end{array}$ & 0.3 & $\begin{array}{l}2.71 \\
(0.1)\end{array}$ & 0.3 & $\begin{array}{l}2.67 \\
(0.1)\end{array}$ & 103 & $\begin{array}{l}2.68 \\
(0.1)\end{array}$ & 0.3 & $\begin{array}{l}2.68 \\
(0.1)\end{array}$ & 0.3 & 2.67 & \\
\hline & opt & - & 2.66 & 0.3 & 2.68 & 0.3 & 2.66 & 0.3 & 2.67 & 03 & 2.6 & 0.4 & 2.62 & 0.4 & 2.61 & \\
\hline & opt2 & _ & .82 & 0.2 & 2.79 & 0.2 & & & & & 2.80 & 0.2 & 2.82 & 0.2 & 2.82 & 0. \\
\hline \multirow[t]{3}{*}{$\mathrm{d} 5$} & d & & 13 & 0.3 & $\begin{array}{l}4.93 \\
(0.4)\end{array}$ & 1.1 & 6) & 0 & 7) & 7 & $\begin{array}{l}5.66 \\
(0.5)\end{array}$ & 1.8 & $\begin{array}{l}3.81 \\
(0.3)\end{array}$ & 0.0 & $\begin{array}{l}4.69 \\
(0.4)\end{array}$ & \\
\hline & opt & - & 6 & 0.2 & 4.12 & 0.3 & 3.96 & 0.1 & 4.05 & 0.2 & 3.85 & .0 & 3.79 & 0.0 & 3.78 & \\
\hline & opt2 & - & 16 & 0.3 & 3.95 & 0.1 & & & & & 4.02 & 0.2 & 4.04 & 0.2 & 4.15 & \\
\hline \multirow[t]{3}{*}{$\mathrm{d} 6$} & $\mathrm{md}$ & 3.98 & $\begin{array}{l}3.91 \\
(0.3)\end{array}$ & 0.1 & $\begin{array}{l}4.96 \\
(0.4)\end{array}$ & 1.0 & $\begin{array}{l}4.62 \\
(0.4)\end{array}$ & 0.6 & $\begin{array}{l}4.14 \\
(0.3)\end{array}$ & 0.2 & $\begin{array}{l}4.50 \\
(0.4)\end{array}$ & 0.5 & $\begin{array}{l}3.98 \\
(0.3)\end{array}$ & 0.0 & $\begin{array}{l}4.44 \\
(0.4)\end{array}$ & \\
\hline & opt & - & & 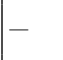 & 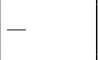 & 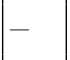 & . & 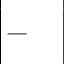 & - & 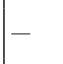 & T. & - & - & - & 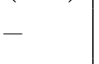 & - \\
\hline & opt2 & & & - & - & 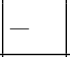 & & 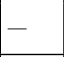 & 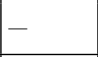 & & & 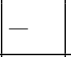 & - & - & 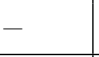 & \\
\hline \multirow[t]{3}{*}{$\mathrm{d} 7$} & $\mathrm{~d}$ & 3.91 & $\begin{array}{c}3.98 \\
(0.3)\end{array}$ & 0.1 & $\begin{array}{l}3.78 \\
(0.2)\end{array}$ & 0.1 & $\begin{array}{l}3.80 \\
(0.2)\end{array}$ & 1 & $\begin{array}{l}34 \\
3)\end{array}$ & 0.0 & $\begin{array}{l}3.99 \\
(0.3)\end{array}$ & 0.1 & $\begin{array}{l}4.05 \\
(0.3)\end{array}$ & 0.1 & $\begin{array}{l}4.21 \\
(0.3)\end{array}$ & \\
\hline & opt & 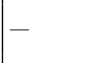 & 3.68 & 0.2 & 3.67 & 0.2 & 3.66 & 0.2 & 3.74 & 0.2 & 3.95 & 0.0 & 3.7 & 0.2 & 3.67 & 0.2 \\
\hline & opt2 & - & 70 & 0.2 & 3.73 & 0.2 & & & & & 3.72 & 0. & 3.69 & 0.2 & 3.67 & \\
\hline
\end{tabular}


TABLE S12: Comparison of the distances (in $\AA$ ) from MD and QM/MM simulations with crystal structure 3ST3 (OFF-state). Chromophore is neutral. 'md' denotes structures averaged over equilibrium MD trajectories. 'opt' and 'opt2' denote the QM/MM optimized structures obtained with present protocol and with the protocol from Ref. 1, respectively.

\begin{tabular}{|c|c|c|c|c|c|c|c|c|c|c|c|c|c|c|c|c|}
\hline D & & 3ST3 & $\begin{array}{l}\text { HSE } \\
\text { GLU } \\
\mathrm{P}\end{array}$ & $\Delta$ & $\begin{array}{l}\text { HSD } \\
\text { GLU } \\
\mathrm{P}\end{array}$ & $\Delta$ & $\begin{array}{l}\text { HSE } \\
\text { GLU } \\
\text { P2 }\end{array}$ & $\Delta$ & $\begin{array}{l}\text { HSD } \\
\text { GLU } \\
\text { P2 }\end{array}$ & $\Delta$ & $\begin{array}{l}\text { HSE } \\
\text { GLU }\end{array}$ & $\Delta$ & $\begin{array}{l}\text { HSD } \\
\text { GLU }\end{array}$ & $\Delta$ & $\begin{array}{l}\text { HSP } \\
\text { GLU }\end{array}$ & $\Delta$ \\
\hline \multirow[t]{3}{*}{ d8 } & $\mathrm{md}$ & 2.59 & L.V & - & $\begin{array}{l}3.86 \\
(1.1)\end{array}$ & 1.3 & $\begin{array}{l}10.4 \\
(7.9)\end{array}$ & 7.8 & $\begin{array}{l}2.87 \\
(0.4)\end{array}$ & 0.3 & L.V & - & $\begin{array}{l}2.64 \\
(0.1)\end{array}$ & 0.0 & L.V & - \\
\hline & opt & - & 2.7 & 0.1 & 2.71 & 0.1 & 2.96 & 0.4 & 2.61 & 0.0 & 2.71 & 0.1 & 2.83 & 0.2 & 2.67 & 0.1 \\
\hline & opt2 & - & 2.7 & 0.1 & 2.67 & 0.1 & & & & & 2.63 & 0.0 & 2.66 & 0.1 & 2.68 & 0.1 \\
\hline \multirow[t]{3}{*}{$\mathrm{d} 9$} & $\mathrm{md}$ & 2.64 & L.V & - & $\begin{array}{l}7.28 \\
(0.7)\end{array}$ & 4.6 & $\begin{array}{l}7.38 \\
(8.2)\end{array}$ & 4.7 & $\begin{array}{l}3.97 \\
(0.6)\end{array}$ & 1.3 & L.V & - & $\begin{array}{l}2.80 \\
(0.1)\end{array}$ & 0.2 & L.V & - \\
\hline & opt & - & 2.71 & 0.1 & 4.36 & 1.7 & 2.62 & 0.0 & 2.53 & 0.1 & 2.6 & 0.0 & 2.62 & 0.0 & 2.51 & 0.1 \\
\hline & opt2 & - & 2.65 & 0.0 & 2.62 & 0.0 & & & & & 2.75 & 0.1 & 2.67 & 0.0 & 2.63 & 0.0 \\
\hline \multirow[t]{3}{*}{$\mathrm{d} 10$} & $\mathrm{md}$ & 3.17 & L.V & - & $\begin{array}{l}4.79 \\
(0.7)\end{array}$ & 1.6 & $\begin{array}{l}9.60 \\
(8.3)\end{array}$ & 6.4 & $\begin{array}{l}5.40 \\
(0.3)\end{array}$ & 2.2 & L.V & - & $\begin{array}{l}3.37 \\
(0.4)\end{array}$ & 0.2 & L.V & 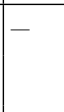 \\
\hline & opt & - & 4.5 & 1.3 & 3.8 & 0.6 & 2.62 & 0.5 & 2.83 & 0.3 & 4.03 & 0.9 & 2.87 & 0.3 & 2.54 & 0.6 \\
\hline & opt2 & - & 2.68 & 0.5 & 3.55 & 0.4 & & & & & 3.44 & 0.3 & 3.13 & 0.0 & 2.75 & 0.4 \\
\hline \multirow[t]{3}{*}{ d11 } & $\mathrm{md}$ & 2.80 & L.V & - & $\begin{array}{l}3.95 \\
(1.4)\end{array}$ & 1.1 & $\begin{array}{l}7.37 \\
(8.6)\end{array}$ & 4.6 & $\begin{array}{l}2.82 \\
(0.2)\end{array}$ & 0.0 & L.V & - & $\begin{array}{l}3.21 \\
(0.4)\end{array}$ & 0.4 & L.V & - \\
\hline & opt & - & - & - & - & - & - & - & - & - & - & - & - & - & - & - \\
\hline & opt2 & - & - & - & - & - & - & - & - & - & - & - & - & - & - & \\
\hline \multirow[t]{3}{*}{$\mathrm{d} 12$} & $\mathrm{md}$ & 2.67 & $\begin{array}{l}4.29 \\
(0.9)\end{array}$ & 1.6 & $\begin{array}{l}4.38 \\
(0.8)\end{array}$ & 1.7 & $\begin{array}{l}4.72 \\
(0.1)\end{array}$ & 2.0 & $\begin{array}{l}4.64 \\
(0.5)\end{array}$ & 2.0 & $\begin{array}{l}2.79 \\
(0.3)\end{array}$ & 0.1 & $\begin{array}{l}3.04 \\
(0.6)\end{array}$ & 0.4 & $\begin{array}{l}2.62 \\
(0.1)\end{array}$ & 0.0 \\
\hline & opt & - & 3.53 & 0.9 & 3.6 & 0.9 & 2.73 & 0.1 & 2.71 & 0.0 & 2.68 & 0.0 & 2.58 & 0.1 & 2.53 & 0.1 \\
\hline & opt2 & - & 2.55 & 0.2 & 2.54 & 0.1 & & & & & 2.68 & 0.0 & 2.65 & 0.0 & 2.65 & 0.0 \\
\hline \multirow[t]{3}{*}{$\mathrm{d} 13$} & md & 2.47 & L.V & - & $\begin{array}{l}3.03 \\
(0.4)\end{array}$ & 0.6 & $\begin{array}{l}2.78 \\
(0.1)\end{array}$ & 0.3 & $\begin{array}{l}2.89 \\
(0.2)\end{array}$ & 0.4 & $\begin{array}{l}4.70 \\
(1.8)\end{array}$ & 2.2 & $\begin{array}{l}4.22 \\
(1.7)\end{array}$ & 1.7 & $\begin{array}{l}2.81 \\
(0.1)\end{array}$ & 0.3 \\
\hline & opt & - & 2.64 & 0.2 & 2.69 & 0.2 & 2.6 & 0.1 & 2.56 & 0.1 & 3.16 & 0.7 & 2.56 & 0.1 & 2.5 & 0.0 \\
\hline & opt2 & - & 2.65 & 0.2 & 2.66 & 0.2 & & & & & 2.60 & 0.2 & 2.58 & 0.1 & 2.56 & 0.1 \\
\hline \multirow[t]{3}{*}{$\mathrm{d} 14$} & $\mathrm{md}$ & 4.88 & $\begin{array}{l}3.74 \\
(1.2)\end{array}$ & 1.1 & $\begin{array}{l}5.18 \\
(0.9)\end{array}$ & 0.3 & $\begin{array}{l}6.56 \\
(0.4)\end{array}$ & 1.7 & $\begin{array}{l}6.36 \\
(0.5)\end{array}$ & 1.5 & $\begin{array}{l}4.52 \\
(0.6)\end{array}$ & 0.4 & $\begin{array}{l}3.69 \\
(1.0)\end{array}$ & 1.2 & $\begin{array}{l}4.59 \\
(0.4)\end{array}$ & 0.3 \\
\hline & opt & - & 6.02 & 1.1 & 5.95 & 1.1 & 4.91 & 0.0 & 5.04 & 0.2 & 5.27 & 0.4 & 4.76 & 0.1 & 4.82 & 0.1 \\
\hline & opt2 & 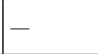 & 4.79 & 0.1 & 4.91 & 0.0 & & & & & 5.01 & 0.1 & 4.95 & 0.1 & 4.93 & 0.0 \\
\hline
\end{tabular}



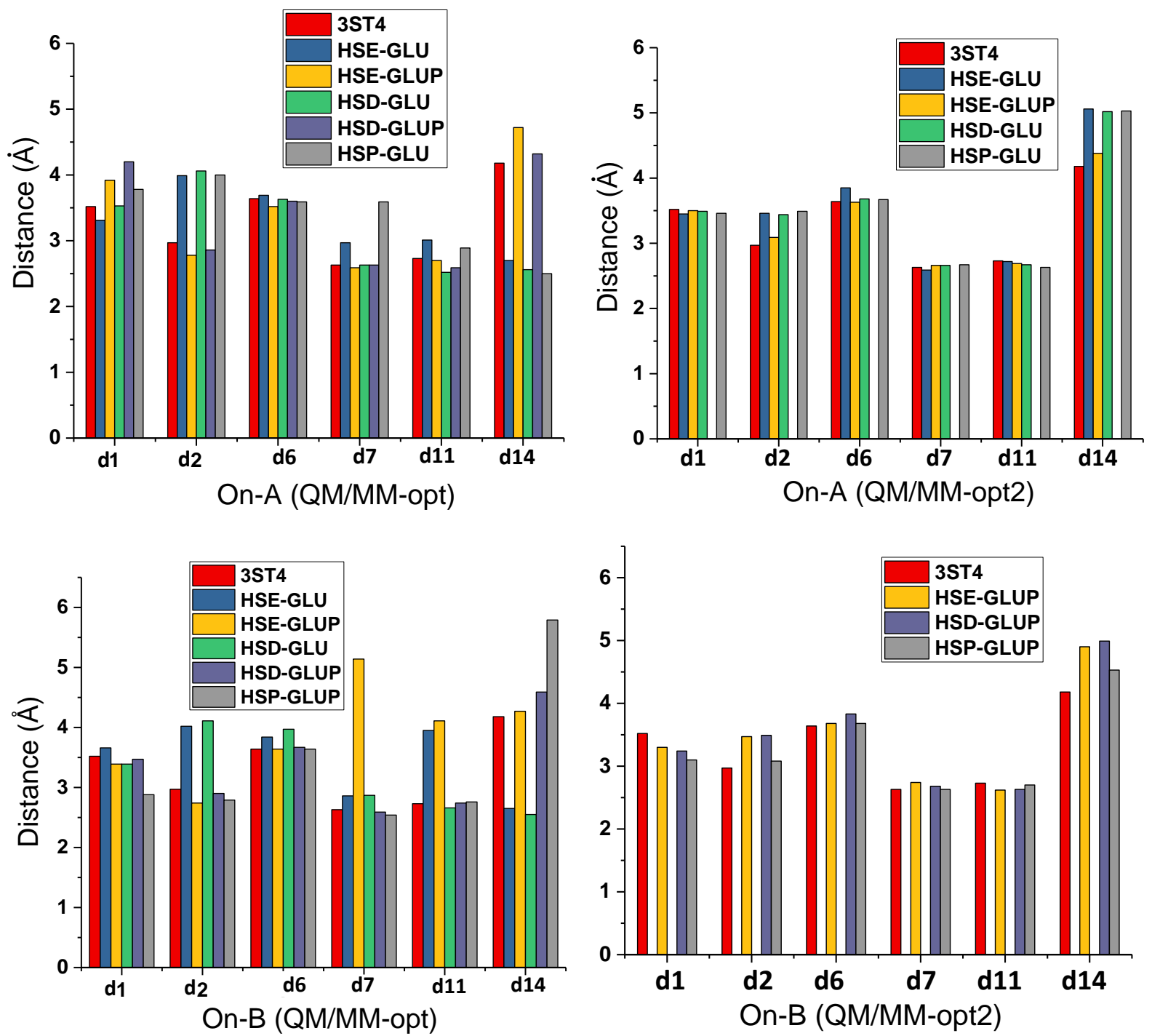

FIG. S4: Key distances for ON-states: Comparison between crystal structure and QM/MM optimization. OPT1 and OPT2 denote two different protocols (see text). See Fig. 5 in the main text for definitions. 

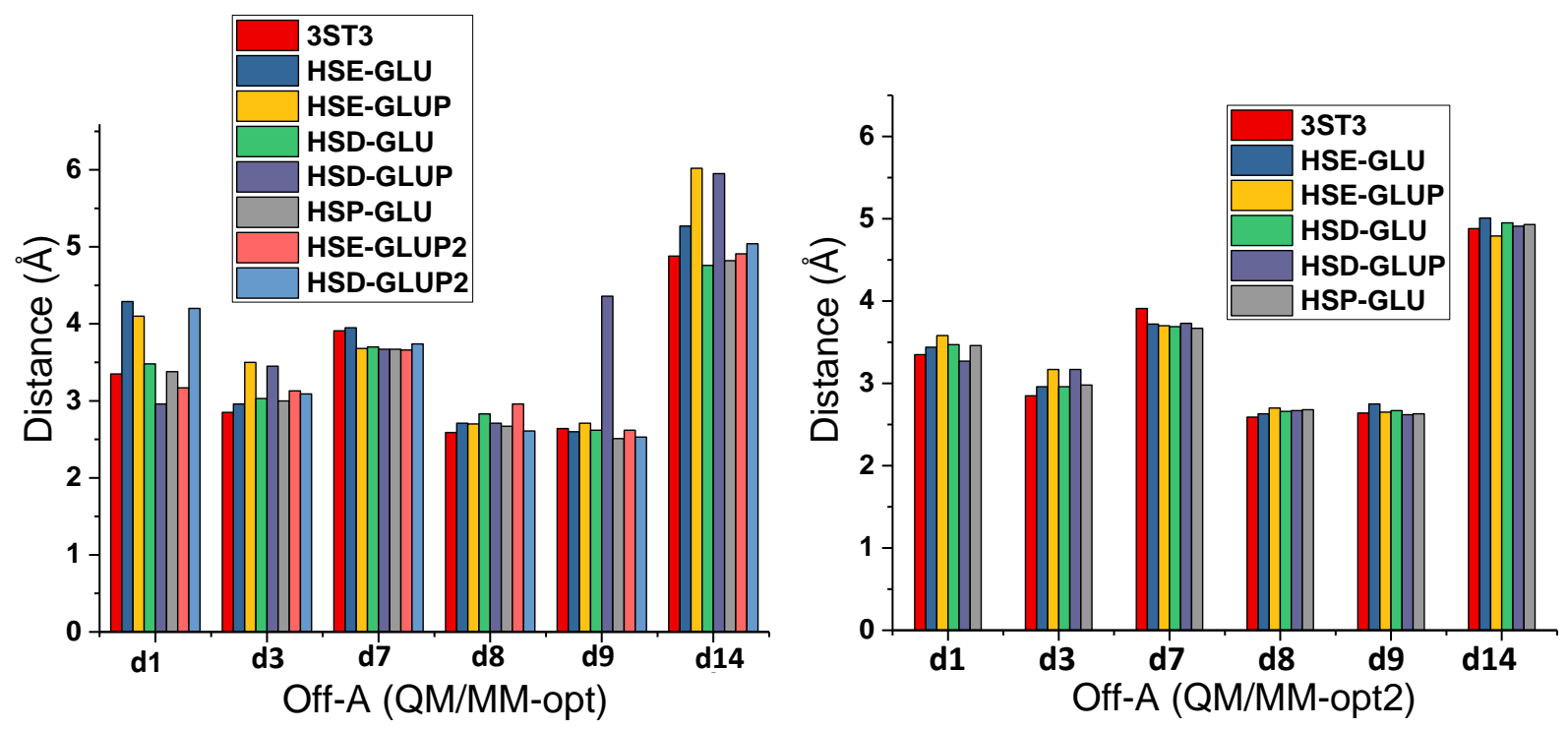

FIG. S5: Key distances for OFF-states: Comparison between crystal structure, average MD values, and QM/MM optimizations. See Fig. 6 of the main text for definitions.
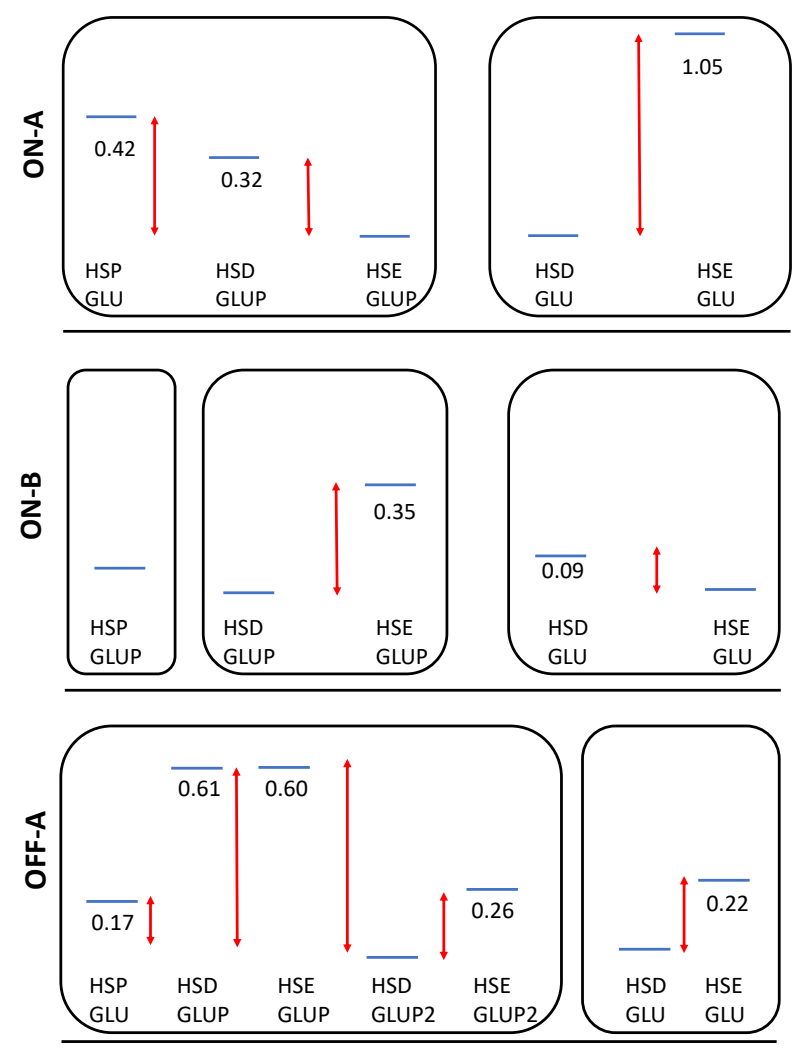

FIG. S6: Energy ordering (eV) of QM/MM (ONIOM) optimized structures (boxes mark the structures with the same number of atoms in QM). 


\section{ANALYSIS OF EXCITED STATES}
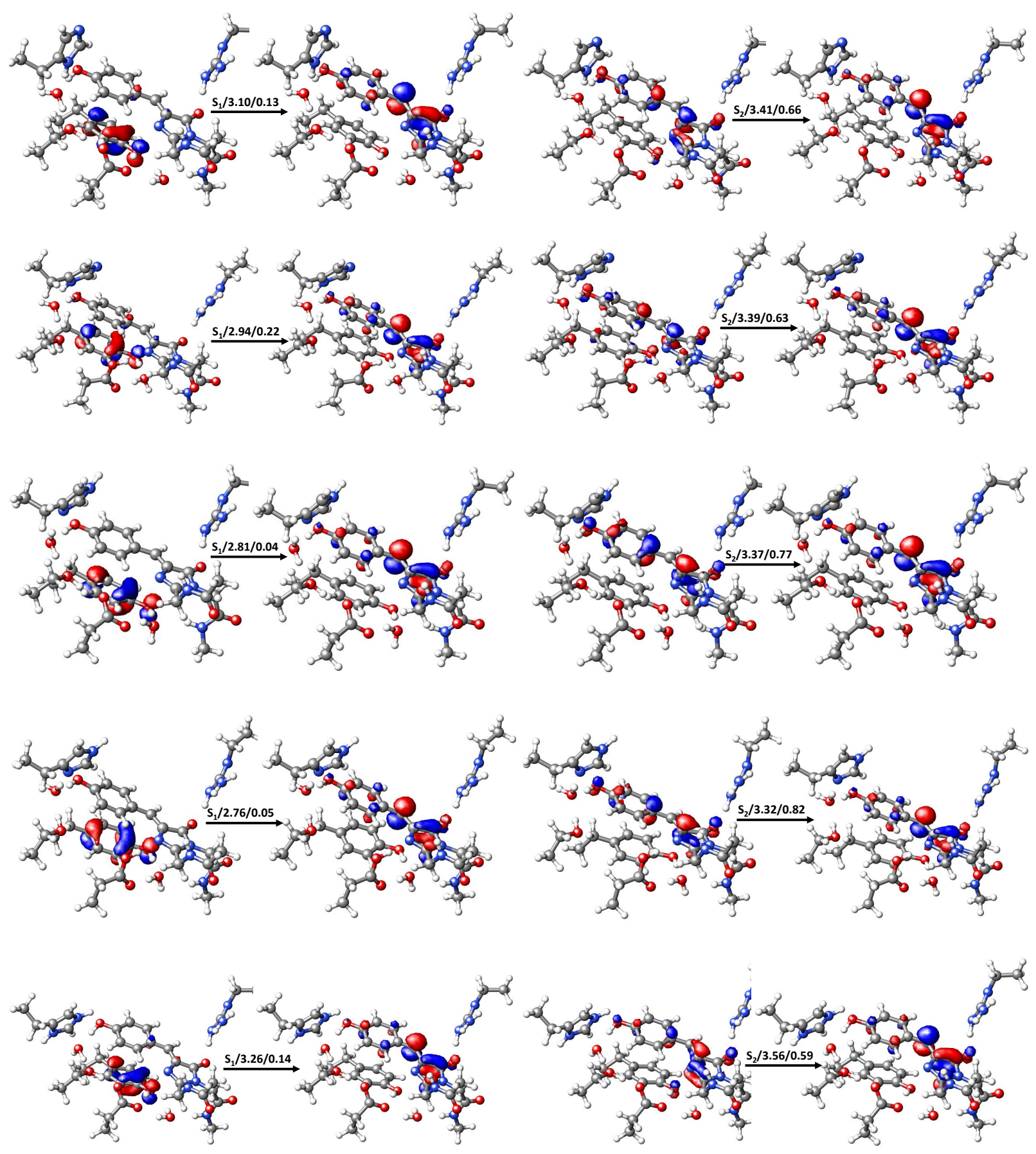

FIG. S7: NTOs of the lowest excited states of the neutral form and different protonation states of His145 and Glu222; TD-DFT, extended QM. Left: CT state; right: LE state; top-to-bottom: HSD-GLU, HSD-GLUP, HSE-GLU, HSE-GLUP, HSP-GLU. 

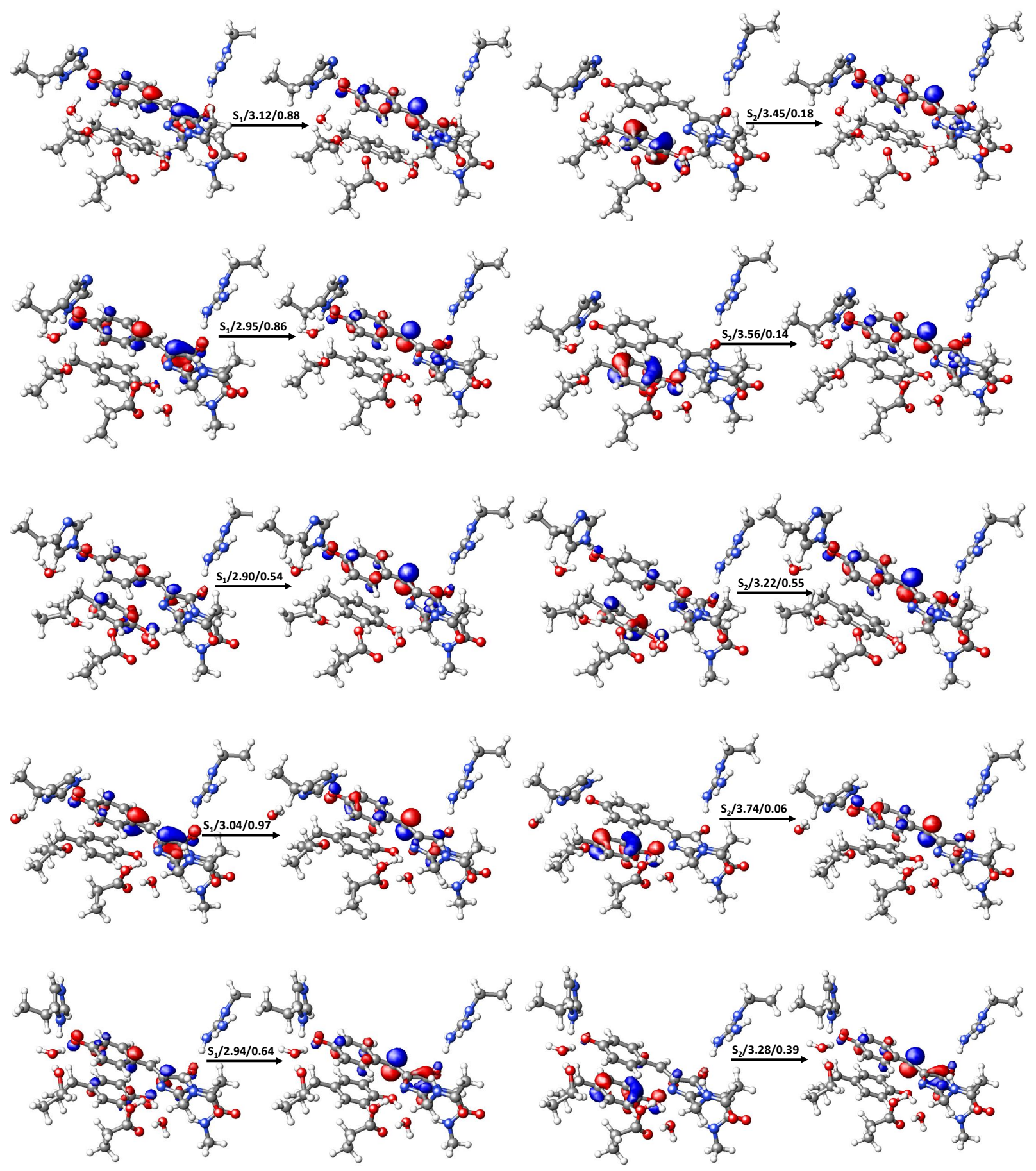

FIG. S8: NTOs of the lowest excited states of the anionic form and different protonation states of His145 and Glu222; TD-DFT, extended QM. Left: LE state; right: CT state; top-to-bottom: HSD-GLU, HSD-GLUP, HSE-GLU, HSE-GLUP, HSP-GLUP. CT state is pushed to much higher energies and disappears in QM/MM calculations. 
TABLE S13: Effect of the protein environment beyond extended QM estimated from the 21 MD snapshots for the neutral chromophore in the ON-state. All energies are in eV; large QM.

\begin{tabular}{|c|c|c|c|c|c|c|}
\hline \multirow[t]{3}{*}{ System } & \multicolumn{2}{|c|}{ State TD-DFT } & \multirow{2}{*}{$\begin{array}{l}\text { TD-DFT } \\
\text { aug-cc-pVDZ }\end{array}$} & \multirow{2}{*}{$\begin{array}{l}\text { TD-DFT } \\
\text { aug-cc-pVDZ }\end{array}$} & \multirow{2}{*}{$\begin{array}{l}\text { TD-DFT } \\
\text { aug-cc-pVDZ }\end{array}$} & \multirow{2}{*}{$\begin{array}{l}\text { TD-DFT } \\
\text { aug-cc-pVDZ }\end{array}$} \\
\hline & & aug-cc-pVDZ & & & & \\
\hline & & $\mathrm{QM} / \mathrm{MM}(\mathrm{MD})$ & QM only (MD) & $\Delta^{a}$ & QM only (opt) ${ }^{b}$ & $\mathrm{QM} / \mathrm{MM}-\operatorname{corr}^{c}$ \\
\hline \multirow[t]{2}{*}{ HSD-GLUP } & $\mathrm{CT}$ & $4.01(0.05)$ & $3.76(0.17)$ & +0.25 & $3.07(0.26)$ & $3.32(0.26)$ \\
\hline & $\mathrm{LE}$ & $3.49(0.56)$ & $3.42(0.52)$ & +0.07 & $3.49(0.54)$ & $3.56(0.54)$ \\
\hline \multirow[t]{2}{*}{ HSE-GLUP } & $\mathrm{CT}$ & $3.96(0.10)$ & $3.70(0.38)$ & +0.26 & $2.88(0.06)$ & $3.14(0.06)$ \\
\hline & $\mathrm{LE}$ & $3.58(0.52)$ & $3.44(0.30)$ & +0.14 & $3.49(0.73)$ & $3.63(0.73)$ \\
\hline \multirow[t]{2}{*}{ HSD-GLU } & $\mathrm{CT}$ & $3.84(0.10)$ & $3.58(0.19)$ & +0.26 & $3.10(0.13)$ & $3.36(0.13)$ \\
\hline & $\mathrm{LE}$ & $3.39(0.49)$ & $3.30(0.51)$ & +0.09 & $3.41(0.66)$ & $3.50(0.66)$ \\
\hline \multirow[t]{2}{*}{ HSE-GLU } & $\mathrm{CT}$ & $3.93(0.08)$ & $3.62(0.11)$ & +0.31 & $2.81(0.04)$ & $3.12(0.04)$ \\
\hline & $\mathrm{LE}$ & $3.45(0.56)$ & $3.37(0.60)$ & +0.08 & $3.37(0.77)$ & $3.45(0.77)$ \\
\hline \multirow[t]{2}{*}{ HSP-GLU } & $\mathrm{CT}$ & $3.88(0.16)$ & $3.71(0.12)$ & +0.17 & $3.26(0.14)$ & $3.43(0.14)$ \\
\hline & LE & $3.56(0.63)$ & $3.53(0.69)$ & +0.03 & $3.56(0.59)$ & $3.59(0.59)$ \\
\hline
\end{tabular}

${ }^{a} \Delta$ is the difference in excitation energies in QM/MM and QM only calculation evaluated using structures from $21 \mathrm{MD}$ snapshots.

${ }^{b}$ QM only excitation energies computed using ONIOM optimized structures.

${ }^{c}$ Extrapolated values: QM only excitation energies computed using ONIOM optimized structures plus $\Delta$ correction. 
TABLE S14: Effect of the protein environment beyond extended QM estimated from the 21 MD snapshots for the anionic chromophore in the ON-state. All energies are in eV; large QM.

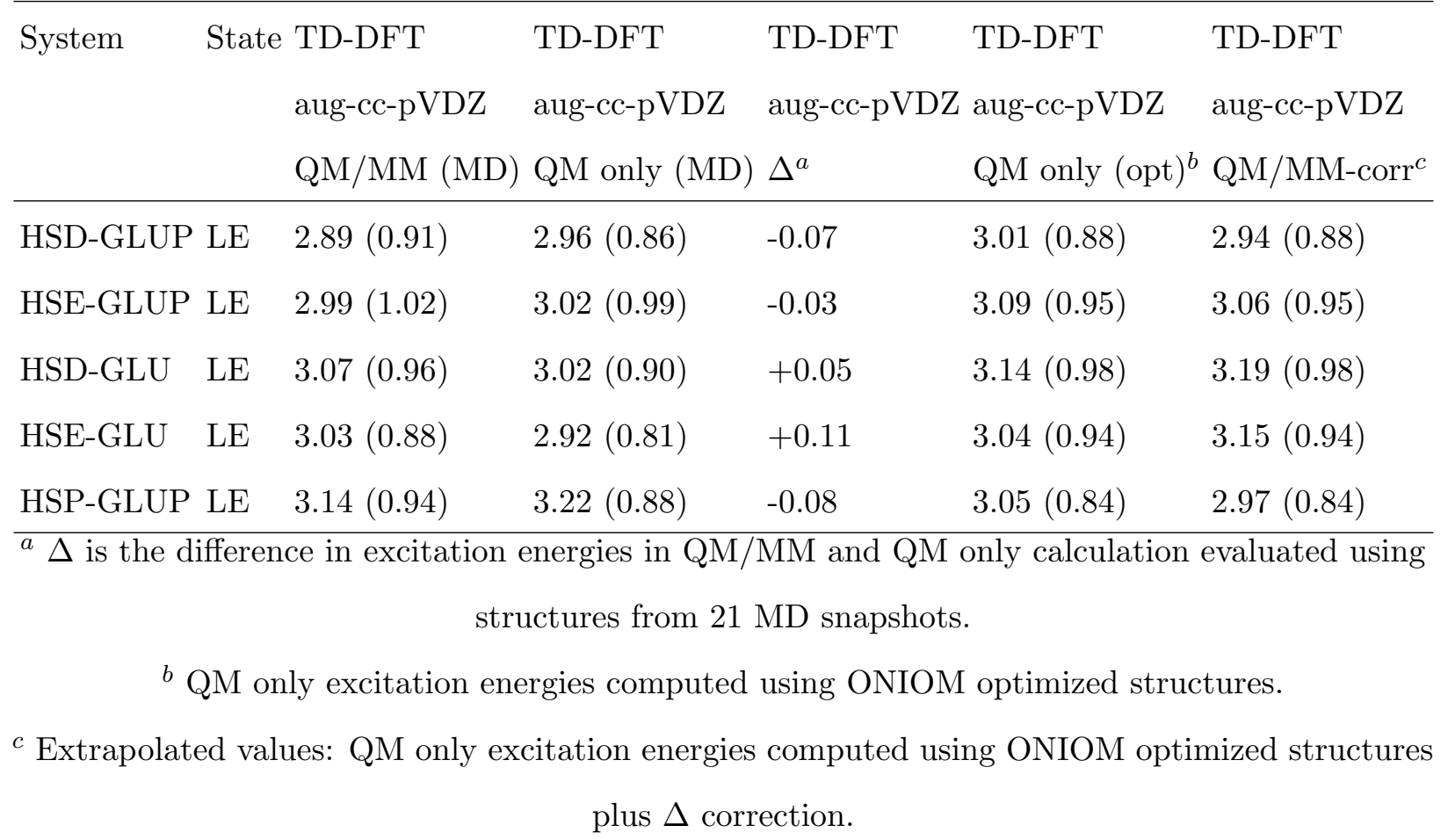


TABLE S15: Effect of the protein environment beyond extended QM estimated from the 21 MD snapshots for the OFF-form (neutral chromophore). All energies are in eV; large QM.

\begin{tabular}{|c|c|c|c|c|c|c|}
\hline System & State & $\begin{array}{l}\text { TD-DFT } \\
\text { aug-cc-pVDZ }\end{array}$ & $\begin{array}{l}\text { TD-DFT } \\
\text { aug-cc-pVDZ }\end{array}$ & $\begin{array}{l}\text { TD-DFT } \\
\text { aug-cc-pVDZ }\end{array}$ & $\begin{array}{l}\text { TD-DFT } \\
\text { aug-cc-pVDZ }\end{array}$ & $\begin{array}{l}\text { TD-DFT } \\
\text { aug-cc-pVDZ }\end{array}$ \\
\hline & & $\mathrm{QM} / \mathrm{MM}(\mathrm{MD})$ & QM only (MD) & $\Delta^{a}$ & QM only $(\mathrm{opt})^{b}$ & $\mathrm{QM} / \mathrm{MM}-\operatorname{corr}^{c}$ \\
\hline HSD-GLUP & LE & $4.10(0.44)$ & $3.97(0.39)$ & +0.13 & $3.86(0.41)$ & $3.99(0.41)$ \\
\hline HSE-GLUP & LE & $3.89(0.51)$ & $3.88(0.48)$ & +0.01 & $3.92(0.33)$ & $3.93(0.33)$ \\
\hline HSD-GLU & $\mathrm{LE}$ & $4.12(0.56)$ & $4.03(0.36)$ & +0.09 & $3.69(0.53)$ & $3.78(0.53)$ \\
\hline HSE-GLU & LE & $3.88(0.40)$ & $3.79(0.41)$ & +0.09 & $3.52(0.57)$ & $3.61(0.57)$ \\
\hline HSP-GLU & LE & $3.89(0.63)$ & $3.85(0.57)$ & +0.04 & $3.85(0.60)$ & $3.89(0.60)$ \\
\hline HSD-GLUP2 & $\mathrm{LE}$ & $3.79(0.66)$ & $3.63(0.59)$ & +0.16 & $3.52(0.64)$ & $3.68(0.64)$ \\
\hline HSE-GLUP2 & $\mathrm{LE}$ & $3.77(0.40)$ & $3.74(0.32)$ & +0.03 & $3.87(0.58)$ & $3.90(0.58)$ \\
\hline
\end{tabular}

${ }^{a} \Delta$ is the difference in excitation energies in QM/MM and QM only calculation evaluated using structures from 21 MD snapshots.

${ }^{b}$ QM only excitation energies computed using ONIOM optimized structures.

${ }^{c}$ Extrapolated values: QM only excitation energies computed using ONIOM optimized structures plus $\Delta$ correction. 
TABLE S16: TD-DFT excitation energies $(\mathrm{eV})$ of the two lowest states of protein-bound neutral chromophore in the ON-state with different basis sets and different size of QM region; oscillator strength is shown in parentheses.

\begin{tabular}{llllll}
\hline System & \multicolumn{2}{c}{ State Extended QM Extended QM Extended QM Large QM } \\
& & cc-pVDZ & mixed basis & \\
& & Qug-cc-pVDZ & aug-cc-pVDZ \\
& QM only & QM only & QM only & QM only \\
\hline HSD-GLUP LE & $3.43(0.72)$ & $3.40(0.61)$ & $3.39(0.63)$ & $3.49(0.54)$ \\
& CT & $2.91(0.16)$ & $2.96(0.24)$ & $2.94(0.22)$ & $3.07(0.26)$ \\
HSE-GLUP & LE & $3.38(0.87)$ & $3.34(0.82)$ & $3.32(0.82)$ & $3.39(0.73)$ \\
& CT & $2.71(0.03)$ & $2.80(0.05)$ & $2.76(0.05)$ & $2.88(0.06)$ \\
HSD-GLU & LE & $3.46(0.73)$ & $3.41(0.65)$ & $3.41(0.66)$ & $3.44(0.60)$ \\
& CT & $3.09(0.08)$ & $3.12(0.14)$ & $3.10(0.13)$ & $3.16(0.11)$ \\
HSE-GLU & LE & $3.42(0.80)$ & $3.38(0.75)$ & $3.37(0.77)$ & $3.48(0.67)$ \\
& CT & $2.74(0.03)$ & $2.85(0.05)$ & $2.81(0.04)$ & $2.88(0.02)$ \\
HSP-GLU & LE & $3.62(0.65)$ & $3.57(0.58)$ & $3.56(0.59)$ & $3.58(0.52)$ \\
& CT & $3.26(0.09)$ & $3.28(0.14)$ & $3.26(0.14)$ & $3.32(0.13)$ \\
\hline
\end{tabular}

${ }^{a}$ mixed basis: aug-cc-pVDZ for the chromophore and tyrosine and cc-pVDZ for rest of QM.

TABLE S17: TD-DFT excitation energies (eV) of the two lowest states of protein-bound anionic chromophore in the ON-state; oscillator strength is shown in parentheses.

\begin{tabular}{|c|c|c|c|c|}
\hline \multirow[t]{3}{*}{ System } & \multirow[t]{3}{*}{ State } & \multicolumn{3}{|c|}{ Extended QM Extended QM Large QM } \\
\hline & & cc-pVDZ & aug-cc-pVDZ & aug-cc-pVDZ \\
\hline & & QM only & QM only & QM only \\
\hline HSD-GLUP & $\mathrm{LE}$ & $3.03(0.86)$ & $2.95(0.86)$ & $3.01(0.88)$ \\
\hline HSE-GLUP & $\mathrm{LE}$ & $3.12(0.98)$ & $3.04(0.97)$ & $3.09(0.95)$ \\
\hline HSD-GLU & $\mathrm{LE}$ & $3.10(0.79)$ & $3.12(0.88)$ & $3.14(0.98)$ \\
\hline HSE-GLU & $\mathrm{LE}$ & $2.89(0.36)$ & $2.90(0.54)$ & $3.04(0.94)$ \\
\hline HSP-GLUP & $\mathrm{LE}$ & $2.96(0.49)$ & $2.94(0.64)$ & $3.05(0.84)$ \\
\hline
\end{tabular}


TABLE S18: TD-DFT excitation energies (eV) of the two lowest states of protein-bound neutral chromophore in the OFF-state; oscillator strength is shown in parentheses.

\begin{tabular}{lcccc}
\hline System & \multicolumn{2}{c}{ State Extended QM Extended QM Large QM } \\
& & cc-pVDZ & aug-cc-pVDZ & aug-cc-pVDZ \\
& & QM only & QM only & QM only \\
\hline HSD-GLUP & LE & $4.03(0.19)$ & $3.86(0.41)$ & $3.80(0.58)$ \\
HSE-GLUP & LE & $3.97(0.63)$ & $3.92(0.33)$ & $3.93(0.56)$ \\
HSD-GLU & LE & $4.07(0.11)$ & $3.69(0.53)$ & $3.70(0.49)$ \\
HSE-GLU & LE & $3.57(0.56)$ & $3.52(0.57)$ & $3.58(0.54)$ \\
HSP-GLU & LE & $3.89(0.58)$ & $3.85(0.60)$ & $3.83(0.52)$ \\
HSD-GLUP2 LE & $3.58(0.64)$ & $3.52(0.64)$ & $3.57(0.60)$ \\
HSE-GLUP2 & LE & $3.95(0.56)$ & $3.87(0.58)$ & $3.83(0.52)$ \\
\hline
\end{tabular}

TABLE S19: Excitation energies (eV) of the two lowest states of protein-bound neutral chromophore in the ON-state; oscillator strength is shown in parentheses. Extended QM.

\begin{tabular}{|c|c|c|c|c|c|c|}
\hline \multirow[t]{4}{*}{ System } & \multirow[t]{4}{*}{ State } & TD-DFT & SOS-CIS(D) & XMCQDPT2 & $\mathrm{XMCQDPT}^{a}$ & XMCQDPT2 \\
\hline & & \multicolumn{3}{|c|}{ aug-cc-pVDZ aug-cc-pVDZ cc-pVDZ } & \multirow[t]{2}{*}{ cc-pVDZ } & aug-cc-pVDZ \\
\hline & & & & & & /cc-pVDZ \\
\hline & & QM only & QM only & QM only & QM only & QM only \\
\hline \multirow[t]{2}{*}{ HSD-GLUP } & $\mathrm{LE}$ & $3.39(0.63)$ & $2.87(1.04)$ & $2.62(0.50)$ & & $2.59(0.59)$ \\
\hline & $\mathrm{CT}$ & $2.94(0.22)$ & $3.40(0.08)$ & $2.98(0.12)$ & & $3.04(0.17)$ \\
\hline \multirow[t]{2}{*}{ HSE-GLUP } & LE & $3.32(0.82)$ & $2.87(0.99)$ & $2.64(0.57)$ & $2.88(0.31)$ & $2.79(0.41)$ \\
\hline & $\mathrm{CT}$ & $2.76(0.05)$ & $3.17(0.13)$ & $2.89(0.23)$ & $3.09(0.05)$ & $2.51(0.28)$ \\
\hline \multirow[t]{2}{*}{ HSD-GLU } & $\mathrm{LE}$ & $3.41(0.66)$ & $3.10(0.97)$ & $2.89(0.72)$ & $2.85(0.40)$ & $2.83(0.24)$ \\
\hline & $\mathrm{CT}$ & $3.10(0.13)$ & $3.68(0.04)$ & $3.01(0.03)$ & $3.12(0.03)$ & $2.76(0.28)$ \\
\hline \multirow[t]{2}{*}{ HSE-GLU } & $\mathrm{LE}$ & $3.37(0.77)$ & $2.98(0.94)$ & $2.70(0.30)$ & $2.74(0.56)$ & $2.87(0.45)$ \\
\hline & $\mathrm{CT}$ & $2.81(0.04)$ & $3.21(0.10)$ & $2.98(0.29)$ & $3.08(0.01)$ & $2.59(0.18)$ \\
\hline \multirow[t]{2}{*}{ HSP-GLU } & $\mathrm{LE}$ & $3.56(0.59)$ & $3.42(0.93)$ & $3.02(0.14)$ & $2.90(0.16)$ & $2.96(0.26)$ \\
\hline & $\mathrm{CT}$ & $3.26(0.14)$ & $4.05(0.04)$ & $3.10(0.15)$ & $2.93(0.02)$ & $2.94(0.11)$ \\
\hline
\end{tabular}

${ }^{a}$ Using structures and QM definition from the old protocol ${ }^{1}$. 
TABLE S20: Excitation energies of the protein-bound anionic chromophore in the ON-state; oscillator strength is shown in parentheses. Extended QM.

\begin{tabular}{|c|c|c|c|c|c|c|}
\hline \multirow[t]{4}{*}{ System } & \multirow[t]{4}{*}{ state } & TD-DFT & SOS-CIS(D) & XMCQDPT2 & $\mathrm{XMCQDPT}^{a}$ & XMCQDPT2 \\
\hline & & \multicolumn{3}{|c|}{ aug-cc-pVDZ aug-cc-pVDZ cc-pVDZ } & \multirow[t]{2}{*}{ cc-pVDZ } & aug-cc-pVDZ \\
\hline & & & & & & /cc-pVDZ \\
\hline & & QM only & QM only & QM only & QM-only & QM-only \\
\hline HSD-GLUP & LE & $2.95(0.86)$ & $2.41(1.29)$ & $2.30(0.94)$ & $2.39(0.89)$ & $2.16(0.87)$ \\
\hline HSE-GLUP & $\mathrm{LE}$ & $3.04(0.97)$ & $2.68(1.41)$ & $2.58(0.96)$ & $2.37(0.93)$ & $2.39(0.98)$ \\
\hline HSD-GLU & $\mathrm{LE}$ & $3.12(0.88)$ & $2.64(1.34)$ & $2.50(0.93)$ & & $2.37(0.92)$ \\
\hline HSE-GLU & $\mathrm{LE}$ & $2.90(0.54)$ & $2.41(1.29)$ & $2.41(0.90)$ & & $2.29(0.92)$ \\
\hline HSP-GLUP & $\mathrm{LE}$ & $2.94(0.64)$ & $2.43(1.23)$ & $2.39(0.84)$ & $2.41(0.85)$ & $2.27(0.81)$ \\
\hline
\end{tabular}

${ }^{a}$ Using structures and QM definition from the old protocol ${ }^{1}$.

TABLE S21: Excitation energies of the protein-bound neutral chromophore in the OFF-state; oscillator strength is shown in parentheses. Extended QM.

\begin{tabular}{llllll}
\hline System & TDDFT & \multicolumn{2}{c}{ SOS-CIS(D) } & XMCQDPT2 XMCQDPT2 & \\
& aug-cc-pVDZ & Xug-cc-pVDZ cc-pVDZ & cc-pVDZ & aug-cc-pVDZ \\
& & & & & /cc-pVDZ \\
& QM only & QM only & QM only & QM-only & QM-only \\
\hline HSD-GLUP & $3.86(0.41)$ & $3.98(0.83)$ & $4.06(0.52)$ & $3.79(0.21)$ & $3.50(0.63)$ \\
HSE-GLUP & $3.92(0.33)$ & $4.17(0.79)$ & $4.11(0.53)$ & $4.00(0.61)$ & $3.67(0.58)$ \\
HSD-GLU & $3.69(0.53)$ & $3.93(0.76)$ & $3.51(0.58)$ & $3.99(0.66)$ & $3.35(0.55)$ \\
HSE-GLU & $3.52(0.57)$ & $3.62(0.80)$ & $3.30(0.63)$ & $3.97(0.43)$ & $3.05(0.55)$ \\
HSP-GLU & $3.85(0.60)$ & $4.11(0.72)$ & $3.60(0.55)$ & $3.94(0.60)$ & $3.46(0.60)$ \\
HSD-GLUP2 $3.52(0.64)$ & $3.53(0.87)$ & $3.34(0.52)$ & & $3.14(0.52)$ \\
HSE-GLUP2 & $3.87(0.58)$ & $4.06(0.78)$ & $3.92(0.21)$ & & $3.59(0.54)$ \\
\hline
\end{tabular}

${ }^{a}$ Using structures and QM definition from the old protocol ${ }^{1}$. 

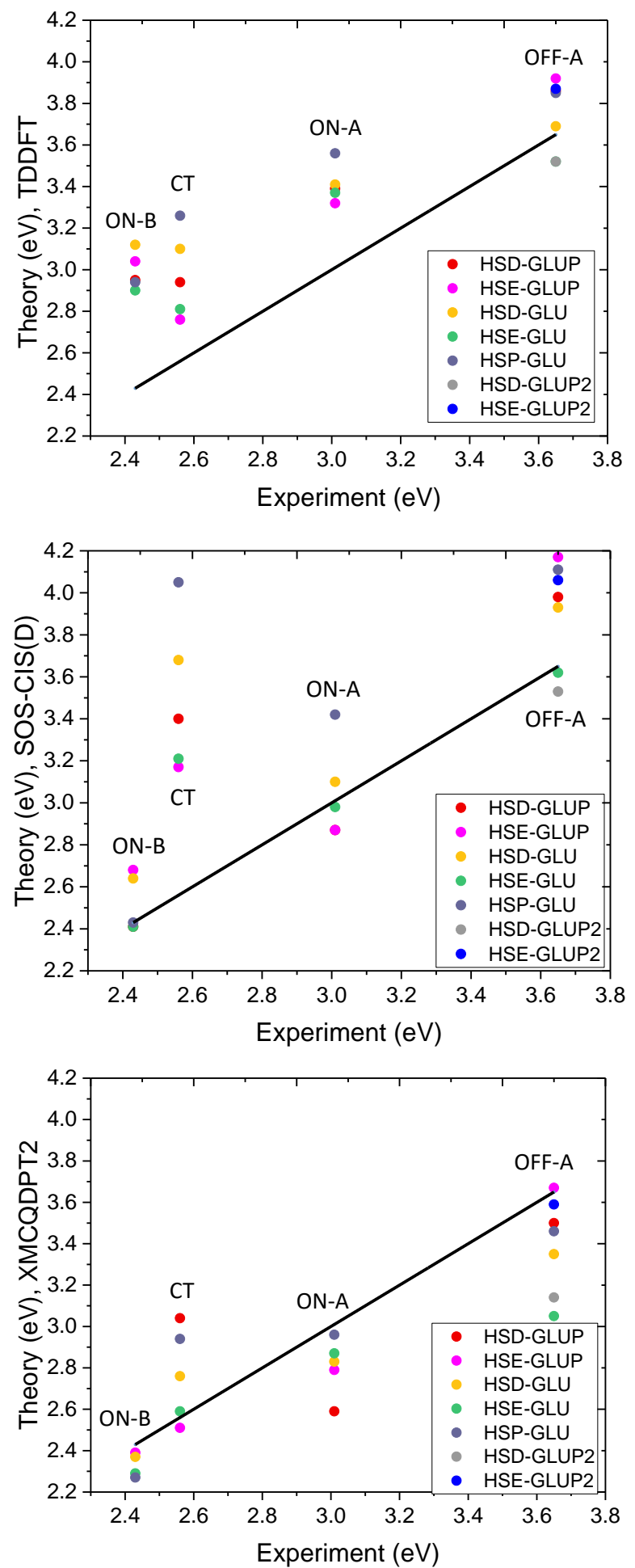

FIG. S9: Excitation energies for different model systems shown against the experimental values. Top: TD-DFT/aug-cc-pVDZ; middle: SOS-CIS(D)/aug-cc-pVDZ; bottom: XMCQDPT2/aug-ccpVDZ/cc-pVDZ. Extended QM. 


\section{STRUCTURES OF POSSIBLE INTERMEDIATES}
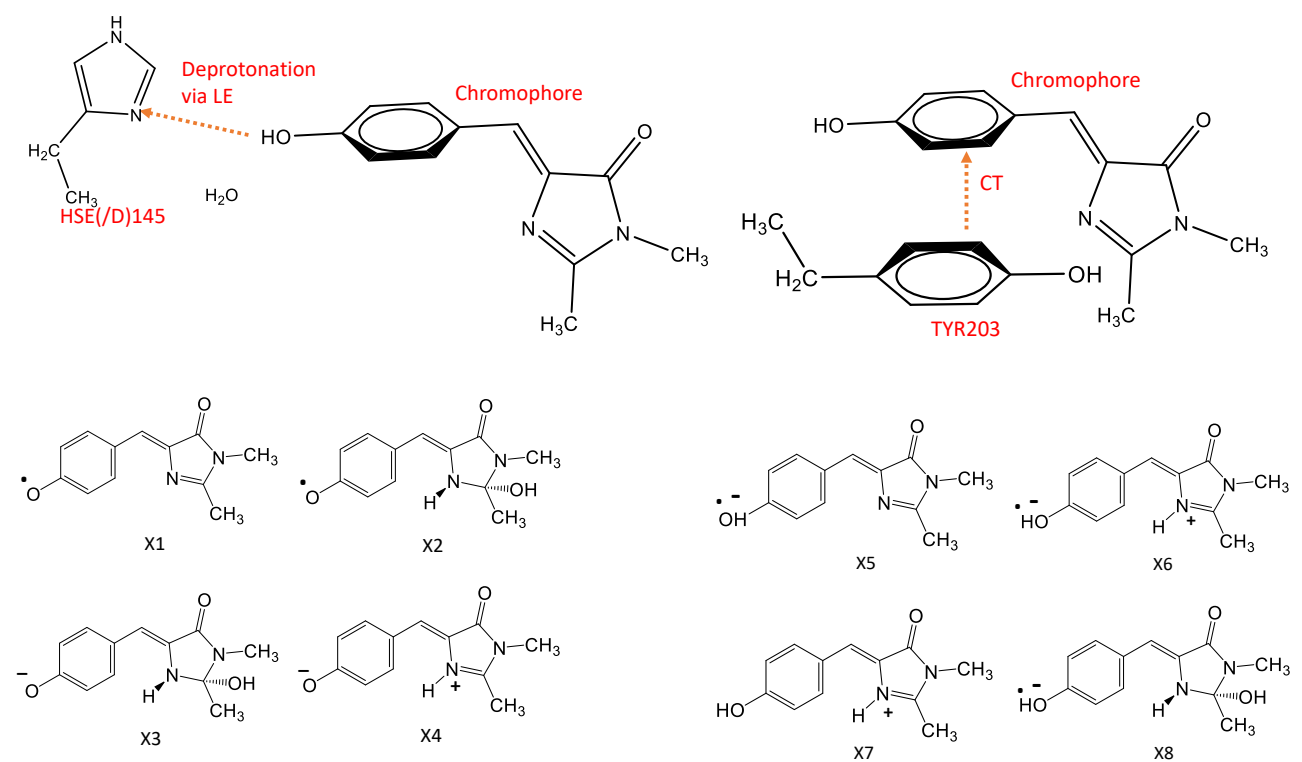

FIG. S10: Two possible initial steps for Dreiklang photoconversion. Ref. 5 proposed that the photoconversion begins by ESPT (left), forming anionic chromophore, which undergoes further transformation. Following this route, one can consider structures X1-X4 as possible candidates for reaction intermediate $\mathrm{X}$. We propose an alternative mechanism via CT state (right). Following this route, one can consider structures $\mathrm{X} 5-\mathrm{X} 8$ as possible candidates for reaction intermediate $\mathrm{X}$.

We considered several structures of the intermediates. Fig. S10 shows 2 possible scenarios for initiating photoconversion. Ref. 5 proposed that the photoconversion begins by ESPT, forming anionic chromophore, which undergoes further transformation. Following this route, one can consider structures X1-X4 as possible candidates for reaction intermediate X. As explained in the main text, there are several major objections to this mechanism. We propose an alternative mechanism via CT state. Following this route, one can consider structures $\mathrm{X} 5-\mathrm{X} 8$ as possible candidates for reaction intermediate $\mathrm{X}$.

Intermediate X5 corresponds to the chromophore in the $\mathrm{CT}$ state $\left(\mathrm{Chro}^{-}\right)$. Intermediate $\mathrm{X} 5$ is the result of proton transfer to $\mathrm{Chro}^{-}$, forming neutral radical. $\mathrm{X} 7$ is the result of the protonated chromophore after back-transfer of the electron. X8 is the result of the hydrated chromophore which still has the extra electron. 


\section{OPTIMIZATION AND AIMD SIMULATIONS: ADDITIONAL RESULTS}

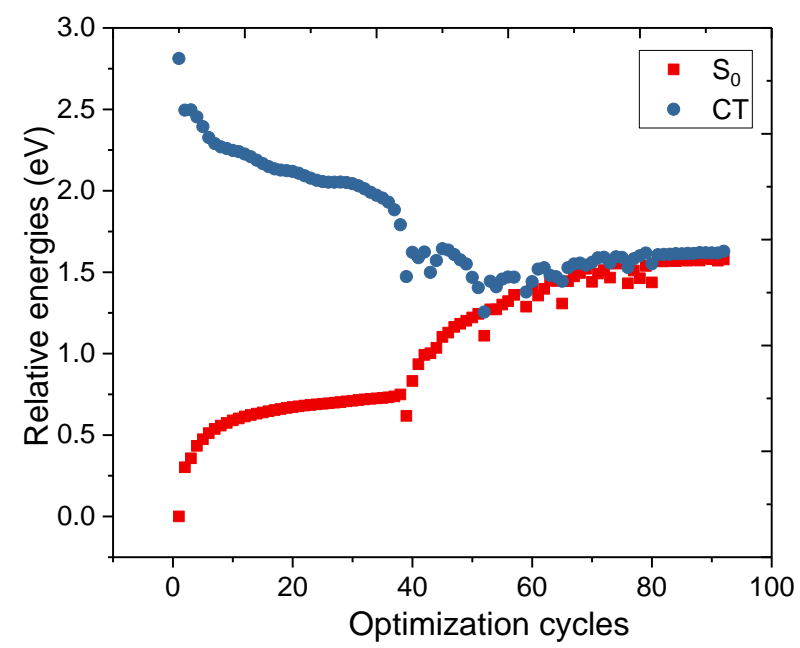

FIG. S11: Energies of the Kohn-Sham reference state $\left(\mathrm{S}_{0}\right)$ and CT state along optimization path (on-A-HSE-GLUP structure).
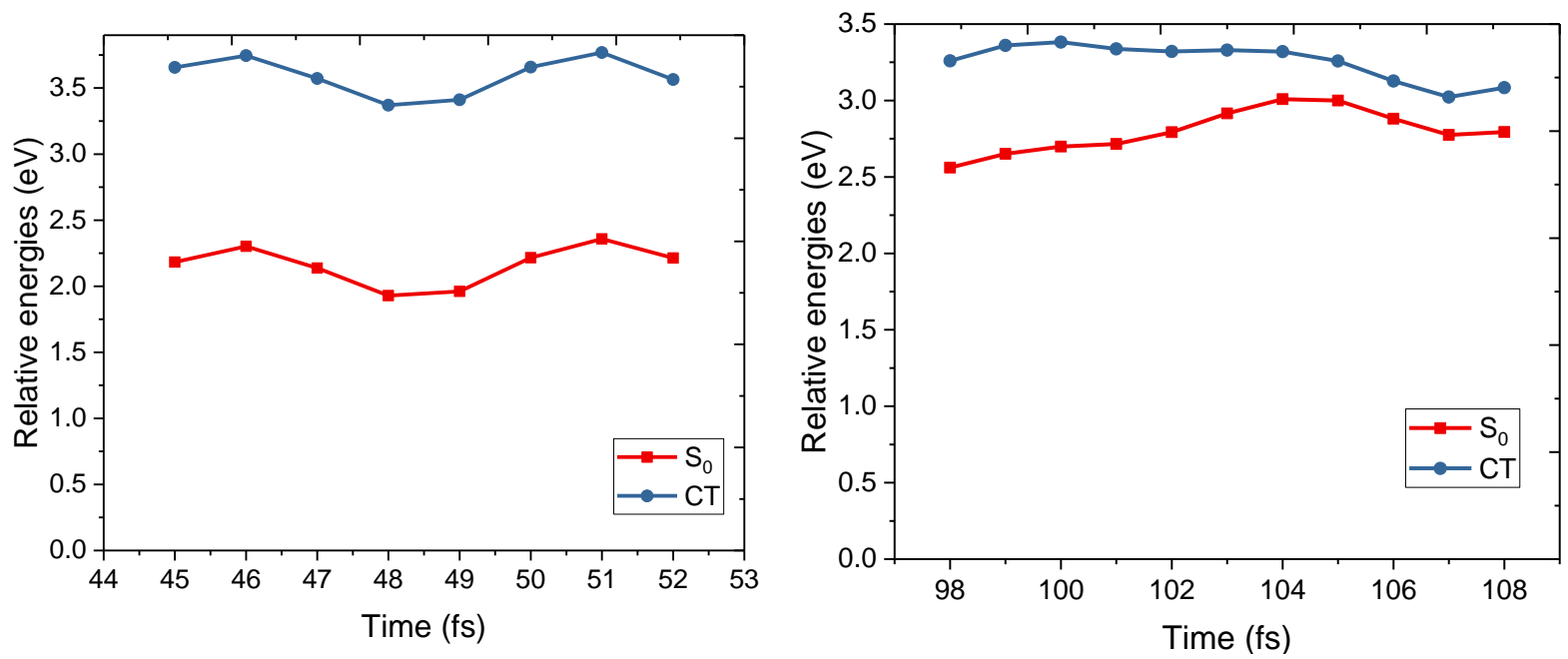

FIG. S12: Ground and excited state during the first two steps of the reaction in CT state (on-AHSE-GLUP structure). Left: 1st step — proton abstraction by chromophore's $\mathrm{N}$ from protonated Glu222. Right: 2nd step — proton transfer from Tyr203 to deprotonated Glu222. 


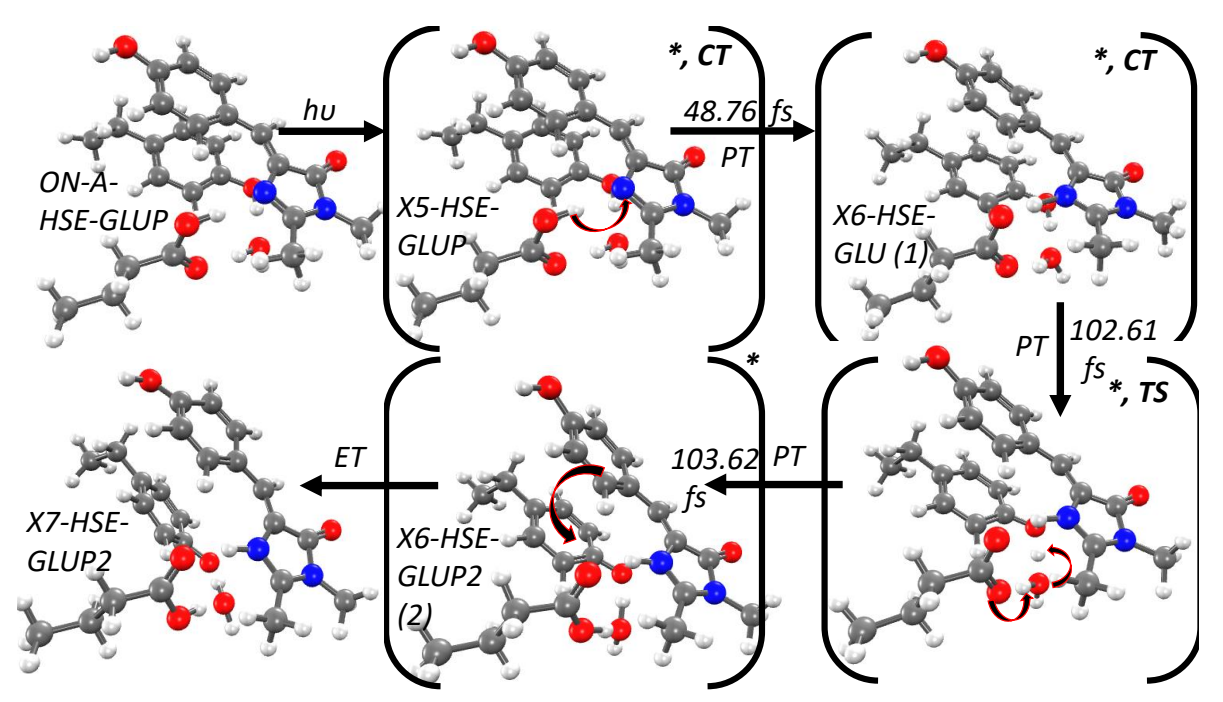

FIG. S13: Analysis of the AIMD trajectory on the CT state (on-A-HSE-GLUP structure).

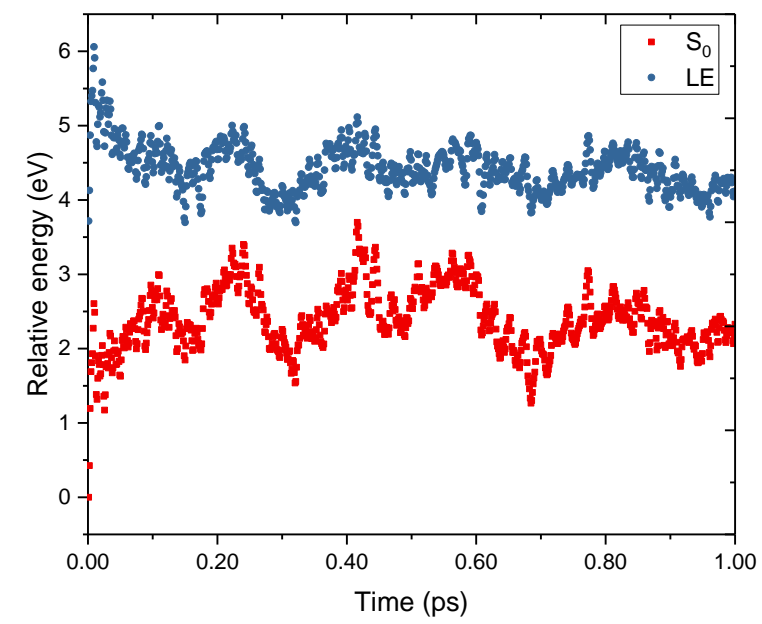

FIG. S14: Energies of the Kohn-Sham reference state $\left(\mathrm{S}_{0}\right)$ and the LE state (2nd TD-DFT state) along the AIMD trajectory on the LE potential energy surface. 


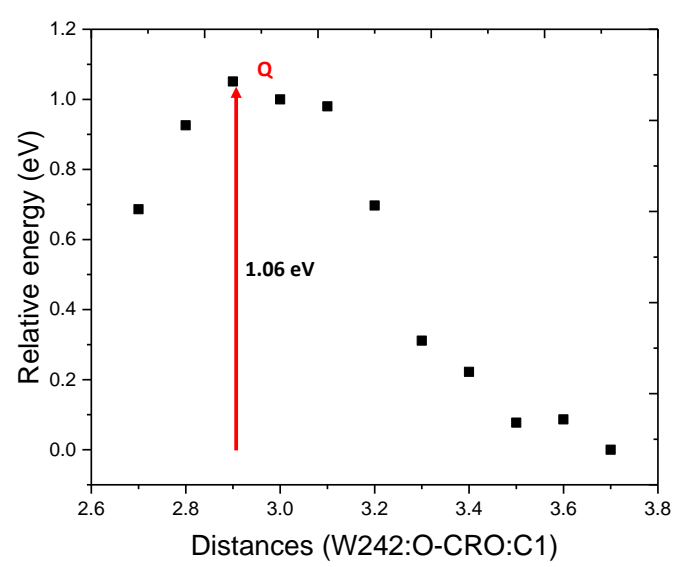

FIG. S15: Relaxed energy profile on the ground state surface (starting from X7 intermediate) along hydration reaction coordinate defined as W242:O-CRO:C1 distance. Zero energy corresponds to the energy of the reference state of the structure at $t=248 \mathrm{fs}$, roughly corresponding to X7. ONIOM, $\omega$ B97X-D/aug-cc-pVDZ/CHARMM27. 
1 B. L. Grigorenko, I. Polyakov, A. I. Krylov, and A. V. Nemukhin, Computational modeling reveals the mechanism of fluorescent state recovery in the reversibly photoswitchable protein Dreiklang, J. Phys. Chem. B 123, 8901 (2019).

2 D.A. Case, T.A. Darden, T.E. Cheetham III, C.L. Simmerling, J. Wang, R.E. Duke, R. Luo, K.M. Merz, B. Wang, D.A. Pearlman, M. Crowley, S. Brozell, V. Tsui, H. Gohlke, J. Mongan, V. Hornak, G. Cui, P. Beroza, C. Schafmeister, J.W. Caldwell, W.S. Ross, and P.A. Kollman, Amber 8, University of California, San Francisco (2004).

3 A. M. Bogdanov, A. Acharya, A. V. Titelmayer, A. V. Mamontova, K. B. Bravaya, A. B. Kolomeisky, K. A. Lukyanov, and A. I. Krylov, Turning on and off photoinduced electron transfer in fluorescent proteins by $\pi$-stacking, halide binding, and Tyr145 mutations, J. Am. Chem. Soc. 138, 4807 (2016).

4 T. Sen, A. V. Mamontova, A. V. Titelmayer, A. M. Shakhov, A. A. Astafiev, A. Acharya, K. A. Lukyanov, A. I. Krylov, and A. M. Bogdanov, Influence of the first chromophore-forming residue on photobleaching and oxidative photoconversion of EGFP and EYFP, Int. J. Mol. Sci. 20, 5229 (2019).

5 F. Lacombat, P. Plaza, M.-A. Plamont, and A. Espagne, Photoinduced chromophore hydration in the fluorescent protein Dreiklang is triggered by ultrafast excited-state proton transfer coupled to a low-frequency vibration, J. Phys. Chem. Lett. 8, 1489 (2017). 


\section{Cartesian coordinates}

\$\omega\$B97x-D/aug-cc-PVDZ optimized geometry of ON-A

$\begin{array}{rrrr}\mathrm{C} & 3.3091392714 & -2.1308271392 & 0.2134303414 \\ \mathrm{C} & 2.5848741735 & -0.8348662376 & 0.1239310625 \\ \mathrm{~N} & 1.2916641099 & -0.7062162216 & 0.0942181618 \\ \mathrm{~N} & 3.2701059046 & 0.3635901151 & 0.0685756524 \\ \mathrm{C} & 2.3357858127 & 1.3982844442 & -0.0209219832 \\ \mathrm{O} & 2.5968716423 & 2.5827704984 & -0.0961886389 \\ \mathrm{C} & 1.0371003294 & 0.6742859464 & 0.0103467948 \\ \mathrm{C} & 4.7015770906 & 0.5603497681 & 0.0765287779 \\ \mathrm{C} & -0.1417695006 & 1.3303127748 & -0.0352202594 \\ \mathrm{C} & -1.5055282597 & 0.8229202531 & 0.0235778174 \\ \mathrm{C} & -1.8302960776 & -0.4988899432 & 0.3730205514 \\ \mathrm{C} & -2.5611520916 & 1.7062617066 & -0.2673764360 \\ \mathrm{C} & -3.1521275670 & -0.9212842253 & 0.4100735937 \\ \mathrm{C} & -3.8848542662 & 1.2940841740 & -0.2387418156 \\ \mathrm{C} & -4.1826582533 & -0.0285745246 & 0.0986161647 \\ \mathrm{O} & -5.4929560483 & -0.3891408228 & 0.1157958982 \\ \mathrm{O} & 0.1390370476 & -3.2637870116 & -0.4682586431 \\ \mathrm{H} & -4.6968740858 & 1.9812772426 & -0.4697851985 \\ \mathrm{H} & -2.3334240442 & 2.7411406013 & -0.5259990750 \\ \mathrm{H} & -1.0460681059 & -1.2037186889 & 0.6327788152 \\ \mathrm{H} & -3.3820937267 & -1.9523109421 & 0.6853201846 \\ \mathrm{H} & -5.5719772007 & -1.3146707430 & 0.3630919490 \\ \mathrm{H} & -0.0367131841 & 2.4135082304 & -0.1372189082 \\ \mathrm{H} & 4.0264013014 & -2.2305455081 & -0.6126829750 \\ \mathrm{H} & 2.5929853801 & -2.9561462493 & 0.1733282001 \\ \mathrm{H} & -0.3313787551 & -3.2391413644 & -1.3043173035 \\ \mathrm{H} & 0.4335890885 & -2.3447530424 & -0.3190811532 \\ \mathrm{H} & 4.8761687873 & 1.6407366986 & 0.0448199919 \\ \mathrm{H} & 5.1486624992 & 0.1481334333 & 0.9898458420 \\ \mathrm{H} & 5.1693592899 & 0.0952169346 & -0.8007436453 \\ \mathrm{H} & 3.8772807657 & -2.1811294827 & 1.1530660628\end{array}$

Nuclear Repulsion Energy $=1148.9571183284$ hartrees

\$\omega\$B97x-D/aug-cc-PVDZ optimized geometry of ON-B

$\begin{array}{lrrr}\mathrm{C} & 3.3027564644 & -2.1451620823 & 0.3323983921 \\ \mathrm{C} & 2.5737735642 & -0.8526074790 & 0.1981290730 \\ \mathrm{~N} & 1.2823706886 & -0.7237066570 & 0.1642001048 \\ \mathrm{~N} & 3.2359788335 & 0.3523460149 & 0.0807602122 \\ \mathrm{C} & 2.2771345443 & 1.3738511326 & -0.0622696898 \\ \mathrm{O} & 2.5628353477 & 2.5659494616 & -0.1987306435 \\ \mathrm{C} & 1.0204788214 & 0.6486532882 & 0.0065441900\end{array}$




$\begin{array}{lrrr}\text { C } & 4.6560018075 & 0.5786771117 & 0.0694292520 \\ \mathrm{C} & -0.2098616682 & 1.2735554584 & -0.0897594095 \\ \mathrm{C} & -1.5267982581 & 0.7854494775 & 0.0152268322 \\ \mathrm{C} & -1.8674931179 & -0.5506343601 & 0.4014120560 \\ \mathrm{C} & -2.6190902147 & 1.6650261570 & -0.2671903500 \\ \mathrm{C} & -3.1656262017 & -0.9737413462 & 0.4658381042 \\ \mathrm{C} & -3.9240287637 & 1.2604938988 & -0.2163276932 \\ \mathrm{C} & -4.2917518616 & -0.1019518288 & 0.1454115231 \\ \mathrm{O} & -5.4766677490 & -0.4910057136 & 0.1929076623 \\ \mathrm{O} & 0.1047780464 & -3.0917160155 & -0.9336598844 \\ \mathrm{H} & -4.7375854834 & 1.9513013766 & -0.4442145162 \\ \mathrm{H} & -2.3866026056 & 2.6979301363 & -0.5431817413 \\ \mathrm{H} & -1.0644472597 & -1.2323274487 & 0.6768919484 \\ \mathrm{H} & -3.4065765254 & -1.9921438854 & 0.7771094494 \\ \mathrm{H} & -0.1144289704 & 2.3457104150 & -0.2899630401 \\ \mathrm{H} & 3.9875292489 & -2.3051938566 & -0.5134348012 \\ \mathrm{H} & 2.5762223272 & -2.9628872630 & 0.3592889813 \\ \mathrm{H} & -0.8208027933 & -2.8936533339 & -1.0978892837 \\ \mathrm{H} & 0.4522007713 & -2.2588002241 & -0.5527228569 \\ \mathrm{H} & 4.8015463229 & 1.6613774886 & -0.0221710796 \\ \mathrm{H} & 5.1247605301 & 0.2267689077 & 0.9996986320 \\ \mathrm{H} & 5.1375312464 & 0.0788741679 & -0.7838656805 \\ \mathrm{H} & 3.9031620573 & -2.1655134287 & 1.2544962406\end{array}$

Nuclear Repulsion Energy $=1133.0413013516$ hartrees

\$\omega\$B97x-D/aug-cc-PVDZ optimized geometry of OFF-A

$\begin{array}{lrrr}\mathrm{C} & -2.4807177970 & 1.0232767613 & 0.1308360114 \\ \mathrm{O} & -2.9575249371 & 1.8995107343 & -0.8723923920 \\ \mathrm{C} & -2.1323269991 & -1.2872645732 & -0.1924974857 \\ \mathrm{~N} & -1.0801849973 & 0.7732152778 & -0.1275990936 \\ \mathrm{O} & -2.3568405148 & -2.4745147945 & -0.3360957613 \\ \mathrm{~N} & -3.0615871950 & -0.3014194133 & -0.0029591808 \\ \mathrm{O} & 5.6031414480 & 0.8439237617 & 0.1268282906 \\ \mathrm{C} & 4.3214475927 & 0.3872898896 & 0.0289212869 \\ \mathrm{C} & -2.7270767761 & 1.6202151601 & 1.5183336256 \\ \mathrm{C} & -0.8105412441 & -0.5940795893 & -0.1659835506 \\ \mathrm{C} & -4.4830424908 & -0.5281887611 & -0.0403433542 \\ \mathrm{C} & 0.3629749230 & -1.2461263359 & -0.1533360145 \\ \mathrm{C} & 2.7692971791 & -1.4085175366 & 0.4296855879 \\ \mathrm{C} & 1.9994783146 & 0.6230982282 & -0.5920715635 \\ \mathrm{C} & 4.0581582691 & -0.8992174398 & 0.5044029532 \\ \mathrm{C} & 3.2901808436 & 1.1436997141 & -0.5279294208 \\ \mathrm{C} & 1.7060014137 & -0.6593237039 & -0.1036964921 \\ \mathrm{H} & -3.7371306959 & 2.3587640496 & -0.5486627594 \\ \mathrm{H} & -0.4270115993 & 1.3692379093 & 0.3632598529\end{array}$




$\begin{array}{rrrr}\text { H } & -2.2163270622 & 2.5879259505 & 1.6002136364 \\ \mathrm{H} & -3.7993064774 & 1.7790480792 & 1.6969912697 \\ \mathrm{H} & -4.6388500521 & -1.5995257282 & -0.2014971224 \\ \mathrm{H} & -4.9602558979 & -0.2389510651 & 0.9070199404 \\ \mathrm{H} & 0.2801677200 & -2.3334025210 & -0.1493499242 \\ \mathrm{H} & 2.5749871045 & -2.4167847654 & 0.7973674554 \\ \mathrm{H} & 1.2225921952 & 1.2130570879 & -1.0773184398 \\ \mathrm{H} & 4.8731698950 & -1.4864630146 & 0.9247161919 \\ \mathrm{H} & 3.4938655846 & 2.1403074589 & -0.9248345398 \\ \mathrm{H} & -4.9438196858 & 0.0293465198 & -0.8673209125 \\ \mathrm{H} & -2.3459991877 & 0.9387961973 & 2.2875916131 \\ \mathrm{H} & 5.6618094344 & 1.7237143586 & -0.2547940897\end{array}$

Nuclear Repulsion Energy $=1177.1217891621$ hartrees

\$\omega\$B97x-D/aug-cc-PVDZ optimized geometry of OFF-B

$\begin{array}{lrrr}\mathrm{C} & -2.4897413522 & 1.0086262888 & -0.0061484603 \\ \mathrm{O} & -3.0275385296 & 1.7979414290 & -1.0703043277 \\ \mathrm{C} & -2.0525438978 & -1.3093135473 & -0.1259711620 \\ \mathrm{~N} & -1.0875483707 & 0.8019122878 & -0.2624201273 \\ \mathrm{O} & -2.2843073959 & -2.5139126969 & -0.1502420472 \\ \mathrm{~N} & -3.0287640951 & -0.3335197974 & -0.0242432060 \\ \mathrm{O} & 5.6437420628 & 0.9036412038 & 0.1513203007 \\ \mathrm{C} & 4.4757966385 & 0.4459307883 & 0.0709872965 \\ \mathrm{C} & -2.7523199588 & 1.7165776518 & 1.3288706891 \\ \mathrm{C} & -0.7829877331 & -0.5872731447 & -0.1813981111 \\ \mathrm{C} & -4.4334712312 & -0.6194164977 & -0.0105042068 \\ \mathrm{C} & 0.4356983231 & -1.1751990408 & -0.0997753525 \\ \mathrm{C} & 2.8580148234 & -1.4145579346 & 0.2863896624 \\ \mathrm{C} & 2.0576852248 & 0.7515375433 & -0.4030158796 \\ \mathrm{C} & 4.1449034165 & -0.9349624289 & 0.3671034123 \\ \mathrm{C} & 3.3398233134 & 1.2505226056 & -0.3380324399 \\ \mathrm{C} & 1.7540355369 & -0.5992302782 & -0.0790305270 \\ \mathrm{H} & -3.8520899241 & 2.1909315183 & -0.7717843531 \\ \mathrm{H} & -0.4813016211 & 1.3895094210 & 0.2982754484 \\ \mathrm{H} & -2.2775661617 & 2.7062752679 & 1.3207229991 \\ \mathrm{H} & -3.8288368262 & 1.8487014621 & 1.5114854738 \\ \mathrm{H} & -4.5402788389 & -1.7067517300 & -0.0947766645 \\ \mathrm{H} & -4.9119560171 & -0.2897285010 & 0.9262706319 \\ \mathrm{H} & 0.3762269517 & -2.2613093489 & 0.0094762717 \\ \mathrm{H} & 2.6654085725 & -2.4664161833 & 0.5210224855 \\ \mathrm{H} & 1.2658869689 & 1.4089751653 & -0.7661899369 \\ \mathrm{H} & 4.9664523280 & -1.5933021002 & 0.6576663323 \\ \mathrm{H} & 3.5427232220 & 2.2893019097 & -0.6081927226 \\ \mathrm{H} & -4.9444688612 & -0.1428182141 & -0.8611569444 \\ \mathrm{H} & -2.3322741380 & 1.1189468857 & 2.1468072929\end{array}$


Nuclear Repulsion Energy $=1157.6033747204$ hartrees 